\title{
Breadth of SARS-CoV-2 Neutralization and Protection Induced by a Nanoparticle Vaccine (75
}

\section{characters)}

4 Dapeng $\mathrm{Li}^{1,2}$, David R. Martinez ${ }^{3}$, Alexandra Schäfer ${ }^{3}$, Haiyan Chen $^{1,2}$, Maggie Barr ${ }^{1}$, Laura L.

5 Sutherland ${ }^{1}$, Esther Lee ${ }^{1,2}$, Robert Parks ${ }^{1}$, Dieter Mielke ${ }^{4}$, Whitney Edwards ${ }^{1}$, Amanda Newman $^{1,2}$, Kevin

6 W. Bock ${ }^{5}$, Mahnaz Minai ${ }^{5}$, Bianca M. Nagata ${ }^{5}$, Matthew Gagne ${ }^{6}$, Daniel C. Douek ${ }^{6}$, C. Todd DeMarco ${ }^{1,2}$,

7 Thomas N. Denny ${ }^{1,2}$, Thomas H. Oguin III $^{1,2}$, Alecia Brown ${ }^{1,2}$, Wes Rountree ${ }^{1,2}$, Yunfei Wang ${ }^{1,2}$,

8 Katayoun Mansouri ${ }^{1}$, Robert J. Edwards ${ }^{1,2}$, Guido Ferrari ${ }^{4}$, Gregory D. Sempowski ${ }^{1,2}$, Amanda Eaton ${ }^{1,4}$,

9 Juanjie Tang ${ }^{7}$, Derek W. Cain ${ }^{1,2}$, Sampa Santra ${ }^{8}$, Norbert Pardi ${ }^{9}$, Drew Weissman ${ }^{10}$, Mark A. Tomai ${ }^{11}$,

10 Christopher B. Fox ${ }^{12}$, Ian N. Moore ${ }^{5}$, Hanne Andersen ${ }^{13}$, Mark G. Lewis ${ }^{13}$, Hana Golding ${ }^{7}$, Robert Seder ${ }^{6}$,

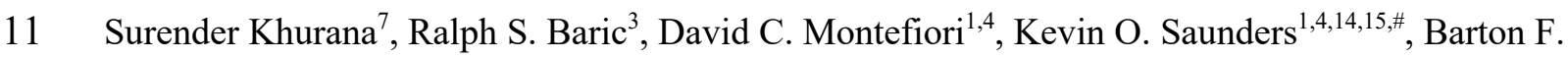

12 Haynes ${ }^{1,2,14, \#}$

$14{ }^{1}$ Duke Human Vaccine Institute, Duke University School of Medicine, Durham, NC 27710, USA.

$15{ }^{2}$ Department of Medicine, Duke University School of Medicine, Durham, NC 27710, USA.

$16{ }^{3}$ Department of Epidemiology, University of North Carolina at Chapel Hill, Chapel Hill, NC 27599, USA.

$17{ }^{4}$ Department of Surgery, Duke University School of Medicine, Durham, NC 27710, USA.

$18{ }^{5}$ Infectious Disease Pathogenesis Section, Comparative Medicine Branch, National Institute of Allergy

19 and Infectious Diseases, National Institutes of Health, Bethesda, MD 20814, USA.

$20{ }^{6}$ Vaccine Research Center, National Institute of Allergy and Infectious Diseases, National Institutes of

21 Health, Bethesda, MD 20814, USA.

$22 \quad{ }^{7}$ Division of Viral Products, Center for Biologics Evaluation and Research (CBER), Food and Drug

23 Administration, Silver Spring, MD 20871, USA.

$24 \quad{ }^{8}$ Beth Israel Deaconess Medical Center, Boston, MA 02215, USA.

$25{ }^{9}$ Department of Microbiology, University of Pennsylvania, Philadelphia, PA 19104, USA.

$26{ }^{10}$ Department of Medicine, University of Pennsylvania, Philadelphia, PA 19104, USA. 
$27{ }^{11}$ Corporate Research Materials Lab, 3M Company, St Paul, MN 55144, USA.

$28{ }^{12}$ Infectious Disease Research Institute, Seattle, WA 98104, USA.

$29{ }^{13}$ BIOQUAL, Rockville, MD 20850, USA.

$30 \quad{ }^{14}$ Department of Immunology, Duke University School of Medicine, Durham, NC 27710, USA.

$31{ }^{15}$ Department of Molecular Genetics and Microbiology, Duke University School of Medicine, Durham,

32 NC 27710, USA.

$33{ }^{\#}$ Correspondence: kevin.saunders@duke.edu (K.O.S.); barton.haynes@duke.edu (B.F.H.)

34 


\section{ABSTRACT (171 words)}

Coronavirus vaccines that are highly effective against SARS-CoV-2 variants are needed to control

37 the current pandemic. We previously reported a receptor-binding domain (RBD) sortase A-conjugated

38 ferritin nanoparticle (RBD-scNP) vaccine that induced neutralizing antibodies against SARS-CoV-2 and

39 pre-emergent sarbecoviruses and protected monkeys from SARS-CoV-2 WA-1 infection. Here, we

40 demonstrate SARS-CoV-2 RBD-scNP immunization induces potent neutralizing antibodies in non-human

41 primates (NHPs) against all eight SARS-CoV-2 variants tested including the Beta, Delta, and Omicron

42 variants. The Omicron variant was neutralized by RBD-scNP-induced serum antibodies with a mean of

43 10.6-fold reduction of ID $_{50}$ titers compared to SARS-CoV-2 D614G. Immunization with RBD-scNPs

44 protected NHPs from SARS-CoV-2 WA-1, Beta, and Delta variant challenge, and protected mice from

45 challenges of SARS-CoV-2 Beta variant and two other heterologous sarbecoviruses. These results

46 demonstrate the ability of RBD-scNPs to induce broad neutralization of SARS-CoV-2 variants and to

47 protect NHPs and mice from multiple different SARS-related viruses. Such a vaccine could provide the

48 needed immunity to slow the spread of and reduce disease caused by SARS-CoV-2 variants such as Delta

49 and Omicron.

51 KEYWORDS

52 SARS-CoV-2, Vaccine, Nanoparticle, Neutralizing Antibodies, Protection, Non-human primates 


\section{INTRODUCTION}

Despite the remarkable success of approved COVID-19 vaccines, additional broadly protective vaccines may be needed to combat breakthrough infections caused by emerging SARS-CoV-2 variants and waning immunity. Moreover, pan-Sarbecovirus vaccines are needed for the prevention of new animal SARS-like viruses that may jump to humans in the future (Levin et al., 2021). Modified mRNA vaccines encapsulated in lipid nanoparticles (LNPs) have proved transformative for COVID-19 vaccine development and for vaccine development in general (Chaudhary et al., 2021; Pardi et al., 2018; Pardi et al., 2020). Developed in 11 months and providing $>90 \%$ efficacy from transmission, the mRNA-1273 and the BNT162b2 COVID-1 vaccines, while showing the most reduction in efficacy from SARS-CoV-2 Beta and Omicron variants, continue to provide significant protection from serious COVID-19 disease, hospitalization, and death (Baden et al., 2021; Polack et al., 2020). The Omicron variant, however, has proved to be more transmissible than previous variants, now accounting for the majority of global isolates (http://www.gisaid.org/hcov19-variants). Likely arising from immunocompromised individuals in South Africa, the Omicron variant spike protein contains 30 mutations compared to the WA-1 strain, and continues to evolve (Wang and Cheng, 2021). While likely less pathogenic than Delta and other SARSCoV-2 variants, the enhanced transmissibility of Omicron, coupled with the sheer number of resulting cases, has resulted in a higher absolute number of COVID-19 patients compared to previous variant

72 infections, thus providing a continued burden on global health care systems.

We previously reported an RBD-based, sortase A-conjugated nanoparticle (RBD-scNP) vaccine

74 formulated with the TLR7/8 agonist 3M-052-aqueous formulation (AF) (hereafter 3M-052-AF) plus

75 Alum, that elicited cross-neutralizing antibody responses against SARS-CoV-2 and other sarbecoviruses,

76 and protected against the WA-1 SARS-CoV-2 strain in non-human primates (NHPs) (Saunders et al.,

77 2021). Here, we found RBD-scNPs induced antibodies that neutralized all variants tested including Beta

78 and Omicron, and protected against Beta and Delta variant challenges in macaques. Moreover, RBD-

79 scNP immunization protected in highly susceptible aged mouse models against challenges of SARS-CoV-

802 Beta variant and other sarbecoviruses. In addition, while formulating RBD-scNP with Alum, 3M-052- 
$81 \mathrm{AF}$, or 3M-052-AF + Alum each protected animals from WA-1 challenge, the 3M-052-AF/ RBD-scNP

82 formulation was optimal for induction of neutralization titers to variants and protection from lung

83 inflammation. Finally, we found that RBD-, N-terminal domain (NTD)- and spike-2P (S2P)-scNPs each

84 protected comparably in the upper and lower airways from WA-1, but boosting with the NTD-scNP

85 protected less well than RBD-scNP or S2P-scNP.

\section{RESULTS}

RBD-scNPs induce neutralizing antibodies against SARS-CoV-2 B.1.1.529 (Omicron) and other variants

RBD-scNPs were used to immunize macaques 3 times four weeks apart (Figure 1A). To test whether RBD-scNP-induced antibodies can neutralize SARS-CoV-2 variants, we collected macaque plasma samples two weeks after the $3^{\text {rd }}$ RBD-scNP immunization (Saunders et al., 2021) and assessed their ability to neutralize pseudovirus infection of 293T-ACE2-TMPRSS2 cells by SARS-CoV-2 WA-1 and 8 variants (Figure 1A). RBD-scNP induced potent plasma neutralizing antibodies against the WA-1 strain

95 with geometric mean titer (GMT) ID $\mathrm{ID}_{50}$ of $12,266.7$, while reduced $\mathrm{ID}_{50}$ titers were observed to different

96 extents for the variants (Figure $1 B-C$ ). In particular, the highest reduction of neutralizing activity was

97 observed for the B.1.351 (Beta) variant, which ranged from 4.1- to 10.2- fold (Figure 1C). To determine

98 if the B.1.1.529 (Omicron) variant could escape RBD-scNP-induced neutralizing antibodies, we

99 compared immune sera for capacity to neutralize the D614G and Omicron variants in a 293T-ACE2

100 pseudovirus assay. Serum antibodies induced by three doses of RBD-scNP immunization (Saunders et al.,

$1012021)$ neutralized both the D614G $\left(\mathrm{GMT} \mathrm{ID}_{50}=40,249\right.$, GMT $\left.\operatorname{ID}_{80}=12,053\right)$ and Omicron (GMT

$102 \mathrm{ID}_{50}=3,794$, GMT ID $80=864$ ) pseudoviruses. A 10.6-fold drop in the GMT $\mathrm{ID}_{50}$ and 14.0-fold drop in the

103 GMT ID 80 were observed (Figure 1D). Thus, high titers of neutralizing antibodies against SARS-CoV-2

104 variants, including Omicron, were elicited by the RBD-scNP immunization in macaques.

105

106 RBD-scNPs protect macaques from SARS-CoV-2 WA-1, Beta and Delta challenge 
Next, we determined if two doses of RBD-scNP vaccination could protect NHPs from challenge by SARS-CoV-2 WA-1, Beta or Delta variants. We immunized cynomolgus macaques with two doses of RBD-scNP vaccine, PBS, or adjuvant alone (Figure $2 A$ ). We also immunized a group of macaques with

110 soluble RBD as a comparator to RBD-scNP immunization to test the effects of multimerization. RBD-

111 scNP and soluble RBD monomer immunization elicited similar titers of antibodies binding to SARS-

$112 \mathrm{CoV}-2$ and other CoV spike antigens (Supplementary Figure 1A), which also similarly blocked ACE2-

113 binding on SARS-CoV-2 spike and bat CoV RsSHC014 spike (Supplementary Figure 1B-C). RBD-

114 scNPs and soluble RBD induced similar levels of antibodies targeting the sarbecovirus cross-neutralizing

115 DH0147-epitope (Hastie et al., 2021; Li et al., 2021a; Li et al., 2021b; Martinez et al., 2021a) on SARS-

116 CoV-2 spike as well as on RsSHC014 spike (Supplementary Figure 1B-C). In pseudovirus neutralization

117 assays, the RBD-scNP group exhibited higher titers of neutralizing antibodies than the soluble RBD

118 group against the WA-1, Alpha, Epsilon, Iota, and Delta viruses, with comparable neutralizing titers

119 against Beta, Gamma, and Kappa variants (Figure 2B). In both groups, neutralizing titers for the Beta,

120 Gamma, and Iota variants were reduced compared to WA-1 (Supplementary Figure 1D). In addition,

121 serum antibodies induced by two doses of RBD-scNP immunization exhibited modest neutralization

122 against Omicron pseudovirus $\left(\mathrm{GMT} \mathrm{ID}_{50}=880\right.$, GMT ID $\left.\mathrm{ID}_{80}=280\right)$, with a 63 -fold drop in the GMT $\mathrm{ID}_{50}$ and

123 37-fold drop in the GMT ID 80 compared to D614G neutralization titers (Figure 2C). Thus, RBD-scNP

124 induced higher neutralizing antibodies than soluble RBD monomer for 5 out of 8 SARS-CoV-2 variant

125 pseudoviruses tested. RBD-scNP showed reduction of Omicron neutralization titers compared to the

126 D614G variant after two immunizations, with far less fold-reduction in Omicron neutralization titers after

127 three immunizations (Figure 1D).

128 Two weeks after the second vaccination, macaques ( $\mathrm{n}=5$ per group) were challenged with SARS-

129 CoV-2 WA-1, SARS-CoV-2 B.1.351 (Beta) variant, or SARS-CoV-2 B.1.617.2 (Delta) variant (Figure

$1302 \boldsymbol{A}$ ). In the PBS or adjuvant alone group, high copies of envelope (E) and nucleocapsid (N) gene

131 subgenomic RNA (sgRNA) were detected in both Bronchoalveolar Lavage (BAL) and nasal swab

132 samples collected on day 2 and 4 post-challenge (Figure 2D-F and Supplementary Figure 1E-G). In 
133 contrast, 5 of 5 animals in the RBD-scNP group, and 4 of 5 animals in the soluble RBD group, were

134 completely protected from WA-1 infection, as indicated by no detectable sgRNA in either BAL or nasal

135 swab (Figure 2D). After the SARS-CoV-2 Beta variant challenge, nasal N gene sgRNA was detected in

136 only 1 of 5 of the RBD-scNP immunized monkeys but in 4 of 5 of the soluble RBD immunized monkeys

137 (Figure $2 \boldsymbol{E}$ ). In addition, after the SARS-CoV-2 Delta variant challenge, all animals that received two

138 doses of RBD-scNP immunization showed no detectable sgRNA in BAL or nasal swab samples (Figure

$1392 F)$.

140 Animals were necropsied 4 days after challenge for histopathologic analysis to determine SARS-

141 CoV-2-associated lung inflammation. After WA-1 and Beta variant challenge, lung tissue haematoxylin

142 and eosin (H\&E) staining revealed no difference between groups (Figure $2 \boldsymbol{G}-\boldsymbol{H}$ ). However,

143 immunohistochemistry (IHC) staining showed the presence of SARS-CoV-2 nucleocapsid antigen in the

144 lungs of macaques administered PBS or adjuvant alone, but not in the lungs of RBD-scNP or soluble

145 RBD immunized monkeys (Figure $2 \boldsymbol{G}-\boldsymbol{H}$ ). Thus, while lung inflammation was observed in immunized

146 macaques, two doses of RBD-scNP immunization protected against viral replication of WA-1, the Beta

147 variant, or Delta variant in both lower and upper airways. In addition, RBD-scNP was superior to soluble

148 RBD in terms of protecting from the hard-to-neutralize Beta variant infection in the upper respiratory

149 tract.

151 RBD-scNPs induce protective responses in mice against SARS-CoV-2 Beta variant and other

152 sarbecoviruses

153 To define the protective efficacy of the RBD-scNP vaccination against different sarbecoviruses, we

154 immunized aged mice with two doses of RBD-scNPs, challenged the mice with mouse-adapted SARS-

155 CoV-2 WA-1, SARS-CoV-2 Beta variant, SARS-CoV-1, or bat CoV RsSHC014 (Figure 2I). In the

156 SARS-CoV-2 WA-1 challenge study, RBD-scNP protected mice from weight loss through 4 days post

157 infection (dpi) and protected from viral replication in the lungs (Figure $2 \boldsymbol{J}$ ). Similar protection from

158 weight loss and lung viral replication were observed in the SARS-CoV-2 Beta variant challenged mice 
(Figure 2K); moreover, by 4 dpi mortality was observed in the unimmunized mice group but no RBDscNP-immunized mice died. Mice immunized with RBD-scNP were also protected against weight loss

161 induced by SARS-CoV-1 and showed $~ 3-\log$ lower average PFU titer in lungs compared to adjuvant

162 alone and unimmunized groups (Figure $2 \boldsymbol{L}$ ). Lastly, RBD-scNP immunization conferred protection

163 against RsSHC014 challenge-induced weight loss and resulted in $\sim 2$-log lower average PFU titer than

164 naïve mice (Figure $2 M$ ). Thus, two doses of RBD-scNP immunization elicited protective immune

165 responses against SARS-CoV-2 Beta variant and multiple other sarbecoviruses in aged mouse models.

\section{Adjuvant is required for RBD-scNP induction of potent plasma and mucosal antibody responses}

To optimize adjuvant formulations for the RBD-scNP vaccine, we next formulated the RBD-scNP immunogen with the TLR7/8 agonist 3M-052-AF alone, with aluminum hydroxide (Alum) alone, or with 3M-052-AF adsorbed to Alum (3M-052-AF + Alum). Control groups included NHPs immunized with immunogen alone (RBD-scNP without adjuvant), adjuvant alone (3M-052-AF, Alum, or 3M-052-AF +

172 Alum without immunogen), or PBS alone (Figure 3A). After three immunizations, RBD-scNP alone

173 without adjuvant induced minimal binding antibodies to SARS-CoV-2 and other CoV spike antigens,

174 whereas higher titers of binding antibodies were induced by RBD-scNP formulated with each adjuvant

175 (Supplementary Figure 2A). While all three adjuvant formulations were highly immunogenic, RBD-

176 scNP adjuvanted with 3M-052-AF induced the highest DH1047-blocking plasma antibodies $(p<0.05$;

177 Wilcoxon rank sum exact test; Supplementary Figure 2 B-C). Mucosal antibody levels tended to be

178 comparable for macaques who received RBD-scNP formulated with 3M-052-AF or 3M-052+Alum, with

179 only low titers being seen when Alum was used to adjuvant the RBD-scNP (Supplementary Figure 2D-

$180 \boldsymbol{E})$.

182 Robust neutralizing antibodies and in vivo protection induced by adjuvanted RBD-scNP

183 While the RBD-scNP alone group showed minimal neutralizing antibody titers, the RBD-scNP +

184 3M-052-AF group had remarkably high pseudovirus neutralizing antibody titers against SARS-CoV-2 
WA-1 strain $\left(\mathrm{GMT} \mathrm{ID}_{50}=59,497\right)$. The GMT ID I0 $_{50}$ RBD-scNP $+3 \mathrm{M}-052-\mathrm{AF}+$ Alum and RBD-scNP

+ Alum groups against WA-1 were 12,267 and 12,610, respectively (Figure 3B). Moreover, RBD-scNPs

$187+3 \mathrm{M}-052-\mathrm{AF}$ immunized animals exhibited the highest magnitudes of neutralizing antibodies against

188 each variant we tested (Figure $3 B$ ). Serum antibodies induced by two immunizations of RBD-scNP

189 showed 94-, 31- and 23-fold drop in the Omicron ID 50 GMT compared to D614G neutralization titers for

190 3M-052-AF + Alum, Alum, and 3M-052-AF adjuvant groups respectively (Figure 3C, upper panel),

191 whereas after three immunizations the reduction-fold of Omicron ID $_{50}$ GMT were 10.6-, 13.3-, and 6.3-

192 fold in the 3M-052-AF + Alum, Alum, and 3M-052-AF adjuvated groups, respectively (Figure 3C, lower

193 panel). The most potent neutralizing antibodies against Omicron were observed in the 3M-052-AF-

194 adjuvanted group, showing ID $_{50}$ GMTs of 1,799 after the $2^{\text {nd }}$ dose and 9,515 after the $3^{\text {rd }}$ dose (Figure

$1953 C$ ). Importantly, although the $3^{\text {rd }}$ boost occurred only 1 month after the $2^{\text {nd }}$ boost, it resulted in a 5.3 -fold

196 increase in Omicron titer with 3M-052-AF adjuvant, 6.4-fold increase with Alum, and 6.3-fold increase

197 with 3M-052-AF + Alum (Figure 3C). Thus, a third boost after only 1 month was effective in enhancing

198 neutralization breadth to the Omicron variant. Moreover, the 3M-052-AF adjuvant induced higher

199 Omicron titers than 3M-052-AF + Alum or Alum alone.

200 To compare in vivo protection of RBD-scNP with different adjuvant formulations, cynomolgus

201 macaques were challenged with the WA-1 strain of SARS-CoV-2 three weeks after the third

202 immunization (Figure 3A). Compared to unimmunized monkeys, the adjuvant alone groups exhibited

203 similar or higher levels of $\mathrm{E}$ and $\mathrm{N}$ sgRNA, and the RBD-scNP immunogen alone reduced sgRNA copies

204 by only $\sim 1-2 \operatorname{logs}$ (Figure 3D and Supplementary Figure 2 F). Immunization with either RBD-scNP

205 adjuvanted with 3M-052-AF + Alum or 3M-052-AF conferred robust protection against SARS-CoV-2

206 infection. Macaques in these groups had under-detection-limit or near-baseline levels of sgRNA N and E

207 in both lower and upper respiratory tracts, demonstrating that adjuvant was required for eliciting potent

208 protection from SARS-CoV-2 challenge. RBD-scNP + Alum immunized macaques showed positive E or

$209 \mathrm{~N}$ gene sgRNA in 1 of 5 and 2 of 5 macaques in BAL samples collected on day 2 post-challenge 
210 respectively. By day 4 post-challenge, all RBD-scNP adjuvanted groups showed no detectable sgRNA

211 (Figure 3D and Supplementary Figure 2F).

212 Histologic analysis of lung tissue showed that RBD-scNP + adjuvant and adjuvant only groups had

213 similar inflammation scores (Figure 3E). IHC staining of the lung tissues exhibited high SARS-CoV-2

214 nucleocapsid antigen expression in the unimmunized and adjuvant alone groups. In contrast, 1 of 5 of the

215 RBD-scNP + Alum immunized animals and 2 of 5 of the immunogen alone immunized animals had low

216 level nucleocapsid antigen expression, and no viral antigen was detected in the RBD-scNP plus 3M-052-

$217 \mathrm{AF}+$ Alum or RBD-scNP plus 3M-052-AF immunized animals (Figure 3E). Therefore, the three

218 adjuvants conferred comparable protection against viral replication by day 4 post-challenge, with $3 \mathrm{M}-$

219 052-AF and 3M-052-AF + Alum providing the best reduction in virus replication when adjuvanting

220 RBD-scNP.

RBD-scNP, NTD-scNP, and S2P-scNP vaccines induce both neutralizing and ADCC-mediating antibodies

While $90 \%$ of neutralizing antibodies target the RBD, neutralizing antibodies can target other sites on spike. Thus, we generated scNPs with NTD and S-2P and compared the antibody response elicited by these ferritin nanoparticles to RBD-scNPs (Figure 4A-B and Supplementary Figure 3A-C). Cynomolgus

228 three immunizations, Spike binding, ACE2-blocking, and neutralizing antibody-blocking antibodies were 229 observed in all three groups (Supplementary Figure 3D-F). In the RBD-scNP and S2P-scNP immunized 230 animals, neutralizing antibodies against SARS-CoV-2 D614G pseudovirus were detected after the first 231 dose and were boosted after the second and third dose at week 6 and 10 (Figure 4C). The NTD-scNP 232 induced sera contained IgGs that blocked NTD neutralizing antibody DH1050.1 binding (Supplementary 233 Figure $3 \boldsymbol{E}$ ). However, NTD-scNP-sera post $2^{\text {nd }}$ and $3^{\text {rd }}$ immunization failed to neutralize the SARS-CoV2342 pseudovirus (Figure 4 C) but neutralized live SARS-CoV-2 WA-1 virus in a microneutralization (MN) 235 assay $\left(\mathrm{GMT} \mathrm{ID}_{50}=2,189\right)$ (Figure 4D). Importantly, S2P-scNP induced comparable plasma neutralizing 
antibody titers compared to RBD-scNP against SARS-CoV-2 WA-1 strain and all eight variants tested

237 ( $p>0.05$; Wilcoxon rank sum exact test; Figure 4E). Among the different variants, the Beta variant

238 showed the largest reduction in neutralization $\mathrm{ID}_{50}$ titer (5.0- to 10.9-fold) (Figure $\left.4 \boldsymbol{F}\right)$. S2P-scNP

239 induced neutralizing antibodies against Omicron pseudovirus, with GMT ID 50 of 436 post-2 ${ }^{\text {nd }}$

240 immunization (38-fold reduction) and GMT ID $_{50}$ of 2,754 post- $3^{\text {rd }}$ immunization (7.2-fold reduction)

\section{1 (Figure 4G).}

242 To examine other antibody functions, we examined plasma antibody binding to cell surface-

243 expressed SARS-CoV-2 spike and antibody-dependent cellular cytotoxicity (ADCC). Plasma antibodies

244 induced by three doses of RBD-scNP, NTD-scNP and S2P-scNP vaccination bound to SARS-CoV-2

245 spike on the surface of transfected cells (Supplementary Figure 3G). In a CD107a degranulation-based

246 ADCC assay (Supplementary Figure 3H), plasma antibodies from all three scNP groups mediated

247 CD107a degranulation of human NK cells in the presence of both SARS-CoV-2 spike-transfected cells

248 and SARS-CoV-2-infected cells (Supplementary Figure 3I). Thus, all three scNP vaccines induced

249 antibodies that neutralize SARS-CoV-2 and mediated ADCC.

\section{RBD-scNP, NTD-scNP, and S2P-scNP vaccines protect macaques against SARS-CoV-2 WA-1}

challenge

To determine NTD-scNP and S2P-scNP immunization conferred protection against SARS-CoV-2,

254 we challenged the immunized macaques with SARS-CoV-2 WA-1 strain via the intratracheal and

255 intranasal routes after the $3^{\text {rd }}$ vaccination. Remarkably, all macaques that received RBD-scNP, NTD-scNP

256 or S2P-scNP were fully protected, showing undetectable or near-detection-limit E or N gene sgRNA

257 (Figure 4H and Supplementary Figure 3J). IHC staining of the lung tissues demonstrated high SARS-

$258 \mathrm{CoV}-2$ nucleocapsid protein expression in the control animals, whereas no viral antigen was detected in

259 any of the scNP-immunized animals (Figure 4I). The sgRNA and histopathology data demonstrated that

260 three doses of NTD-scNP or S2P-scNP immunization provided the same in vivo protection as RBD-scNP

261 immunization, preventing SARS-CoV-2 infection in both lower and upper respiratory tracts. 


\section{RBD-scNP, NTD-scNP and S2P-scNP as boosts for mRNA-LNP vaccine elicited various}

We next assessed the efficacy of the RBD-scNP, NTD-scNP and S2P-scNP as boosts in macaques that received two priming doses of mRNA vaccine. Cynomolgus macaques $(n=5)$ were immunized twice with $50 \mu$ g of S-2P-encoding, nucleoside-modified mRNA encapsulated in lipid nanoparticles (S-2P mRNA-LNP), which phenocopies the Pfizer/BioNTech and the Moderna COVID-19 vaccines.

Subsequently, macaques were boosted with RBD-, NTD- or S2P-scNPs (Figure 4J). Plasma antibody binding patterns were similar among the three groups until animals received the scNP boosting

(Supplementary Figure 4A). Plasma antibodies targeting to ACE2-binding site and neutralizing epitopes were detected after the scNP boosting with cross-reactive antibodies in the DH1047 blocking assay being highest after RBD-scNP or S2P-NP boosting (Supplementary Figure 4B-C). BAL and nasal wash mucosal ACE2-blocking and DH1047-blocking activities tended to be low in magnitude in macaques

Figure 4D-E).

Serum neutralizing titers against the WA-1 strain pseudovirus were similar in the RBD-scNP-boosted group $\left(\mathrm{GMT} \mathrm{ID}_{50}=10,912.1\right)$ and S2P-scNP-boosted group $\left(\mathrm{GMT} \mathrm{ID}_{50}=7799.9\right)($ Figure $4 K)$, while the NTD-scNP-boosted group showed significantly lower titers $\left(\mathrm{GMT} \mathrm{ID}_{50}=3229.8 ; p=0.027\right.$, exact Wilcoxon test). The same differences were also observed in other major variants (Figure 4 K). In addition, 281 in the RBD-scNP- and S2P-scNP-boosted groups, reduced ID $_{50}$ titers were mostly seen for the Beta and

282 Gamma variants, whereas in the NTD-scNP-boosted group, Alpha, Beta, Gamma, Delta, Iota and Kappa 283 variants all showed $>5$-fold reduction of $\mathrm{ID}_{50}$ titers (Figure $4 \boldsymbol{L}$ ).

284 Regarding Omicron neutralization, two doses of S2P mRNA-LNP immunization induced 285 neutralizing antibodies to $\mathrm{D} 614 \mathrm{G}$ with $\mathrm{GMT} \mathrm{ID}_{50}$ of 7,814, which dropped 67-fold when testing for

286 Omicron $\left(\mathrm{GMT} \mathrm{ID}_{50}=116\right)$ (Figure $4 M$ ). The RBD-scNP-boost at one month did not increase D614G 287 neutralization titers, but raised Omicron neutralization titers to GMT ID 50 of 374 (Figure 4M). Thus, 
NTD-scNP was an inferior boost of the S-2P mRNA-LNP vaccine compared to RBD- or S2P-scNPs, and the mRNA-LNP prime/RBD-scNP one-month boost showed reduced boosting capacity for neutralizing antibodies against Omicron, suggesting a longer boosting interval will be needed (Gagne et al., 2022).

\section{Protection of mRNA-LNP-primed and scNP-boosted macaques from SARS-CoV-2 challenge}

Macaques that received mRNA-LNP prime and scNP boosts at one month post-mRNA-LNP primes were challenged with SARS-CoV-2 WA-1 strain after boosting. Four of five RBD-scNP-boosted monkeys and four of five of the S2P-scNP-boosted monkeys were completely protected from SARS-

296 CoV-2 infection, showing no detectable $\mathrm{E}$ or $\mathrm{N}$ gene sgRNA in either BAL or nasal swab samples

297 (Figure 4N and Supplementary Figure $4 \boldsymbol{F}$ ). However, in the NTD-scNP boost group, N gene sgRNA

298 was detected in BAL from three of five animals and in nasal swab samples from two of five animals

299 (Figure 4N). Macaques that received mRNA-LNP prime and RBD-scNP boost had the lowest degree of

300 lung inflammation (Figure 4O). In addition, no viral antigen was observed in lung tissues from either of

301 the immunized groups as indicated by IHC staining for SARS-CoV-2 N protein (Figure 4O).

\section{DISCUSSION}

In this study, the SARS-CoV-2 RBD has maintained conserved neutralizing epitopes among Beta,

305 Delta and Omicron variants, despite the up to 15 RBD amino acid changes for the omicron variant

306 relative to the WA-1 strain. Given the neutralization titers that have protected macaques in challenge

307 studies, we speculate the RBD-scNP vaccine would protect against Omicron challenge with similar

308 efficacy as shown here for the Beta variant. While SARS-CoV-2 continues to mutate during the ongoing

309 pandemic, there are conserved RBD neutralizing epitopes among the SARS-CoV-2 variants. This result is

310 supported by studies with a SARS-CoV-2 virus where 20 naturally occurring mutations were introduced

311 into the spike protein, but the resultant virus was still sensitive to vaccine-induced polyclonal antibody

312 responses (Schmidt et al., 2021). For present and future coverage of SARS-CoV-2 variants, it will be

313 critical to induce a polyclonal response that targets conserved sites on the RBD. Monoclonal antibodies 
314 such as S2x259, S2K146 (Cameroni et al., 2021), DH1047 (Li et al., 2021a; Martinez et al., 2021a) and

315 S309 (Pinto et al., 2020) have defined these key conserved sites upon which vaccines can be designed.

316 Adjuvants play essential roles in vaccine formulation to elicit strong protective immune responses

317 (Coffman et al., 2010) and Alum is used in many currently approved vaccines (HogenEsch et al., 2018).

318 Thus, it was encouraging to see that the RBD-NP vaccine was protective in NHPs when adsorbed to

319 Alum. Compared to Alum, 3M-052-AF + Alum demonstrated superior capacities to elicit neutralizing

320 antibodies against SARS-CoV-2 WA-1 live virus when formulated with SARS-CoV-2 RBD trimer in

321 mice but not in rhesus macaques (Routhu et al., 2021). In addition, 3M-052-adjuvanted gp140 Env

322 vaccine augmented neutralizing antibodies against tier $1 \mathrm{~A}$ HIV-1 pseudovirus in rhesus macaques

323 (Kasturi et al., 2020). 3M-052-AF + Alum is in clinical testing of HIV-1 vaccines (NCT04915768 and

324 NCT04177355). Here we found that 3M-052-AF alone-adjuvanted RBD-scNPs induced not only superior

325 systemic and mucosal antibody responses, but also higher titers of neutralizing antibodies than 3M-052-

$326 \mathrm{AF}+$ Alum-adjuvanted vaccine, demonstrating that 3M-052-AF in the absence of Alum is an optimal

327 adjuvant for scNP. One explanation for this difference could be antagonism between Th1-based immune

328 pathways induced by 3M-052 (Smirnov et al., 2011) and Th2-based pathways induced by Alum (Marrack

329 et al., 2009). Another potential explanation is that physicochemical considerations such as particle size or

330 adsorption interactions between Alum and the RBD-scNP antigen and/or 3M-052-AF are impacting

331 vaccine biodistribution, presentation, or cellular processing, thus affecting downstream immune

332 responses. Such interactions are antigen-dependent (Fox et al., 2016), highlighting the importance of

333 optimizing adjuvant formulation for each unique antigen (Fox et al., 2013; HogenEsch et al., 2018).

334 Coronavirus vaccines formulated with Alum have been reported to be associated with enhanced lung

335 inflammation, particularly with killed vaccines (Arvin et al., 2020; Haynes et al., 2020). However, it is

336 important to note that no enhancement of lung inflammation or virus replication was seen with RBD-

337 scNP/Alum formulations. The RBD-scNP + 3M-052-AF group exhibited the highest neutralizing

338 antibody titers and was the only group showing reduced severity of lung inflammation. That RBD-scNPs 
formulated with Alum alone protected monkeys after immunization raises the possibility of its use in pediatric populations.

While the RBD subunit has been shown to protect against SARS-CoV-2 challenge in animal models

342 (Dai et al., 2020; Francica et al., 2021; Pino et al., 2021; Saunders et al., 2021; Yang et al., 2020), the

343 NTD is also an immunodominant region for neutralizing antibodies (Cerutti et al., 2021; Chi et al., 2020;

344 Li et al., 2021a; Martinez et al., 2021b; McCallum et al., 2021; Voysey et al., 2021). However, NTD is

345 the site of multiple mutations and NTD antibody neutralization is, in general, less potent than RBD

346 antibodies. Here, in this study, NTD-scNP-induced serum neutralizing antibodies were detected using a

347 live SARS-CoV-2 virus but not using pseudovirus. The inconsistent neutralization activities of NTD

348 antibodies in different neutralization assays have been previously observed (Chi et al., 2020; Li et al.,

349 2021a). In addition, the NTD-scNP immunization likely induced not only neutralizing, but also non-

350 neutralizing NTD antibodies (Li et al., 2021a). Serum from the NTD-scNP group did have ADCC

351 activity, suggesting that non-neutralizing Fc receptor-mediated antibody activities could have been

352 involved in protection. In this regard, we previously found that a non-neutralizing NTD antibody DH1052

353 provided partial protection after infusion into mice and non-human primates (Li et al., 2021a). Therefore,

354 the complete protection conferred by scNP vaccination could be a result of both neutralizing and non-

355 neutralizing Fc receptor-mediated antibody activities. Moreover, we found that boosting with RBD-scNP

356 or S2P-scNP after S2P mRNA-LNPs priming afforded complete protection for monkeys after WA-1

357 challenge, while NTD-scNP boosting of S2P mRNA-LNPs priming led to incomplete protection. The

358 mechanism of this latter finding is currently under investigation.

359 Our study has several limitations. First, our study did not evaluate the durability of vaccine-induced 360 immune responses and protection against SARS-CoV-2 variants. Second, we did not set up longer time

361 intervals between the second and the third booster vaccination, to mimic 4-6 month boosting interval in

362 humans. Lastly, we challenged the animals with WA-1 strain, the Beta variant and the Delta variant;

363 future in vivo protection studies will be required upon availability of viral stocks of other SARS-CoV-2

364 variants such as the Omicron variant. 

potent protection in NHPs against WA-1, Beta and Delta variants, and that they induce neutralizing antibodies to all SARS-CoV-2 variants tested in vitro. These findings have important implications for virus escape from neutralizing antibody responses and for development of the next generation of COVID19 vaccines.

\section{MATERIALS AND METHODS}

\section{Animals and immunizations}

The study protocol and all veterinarian procedures were approved by the Bioqual IACUC per a memorandum of understanding with the Duke IACUC, and were performed based on standard operating procedures. Macaques studied were housed and maintained in an Association for Assessment and Accreditation of Laboratory Animal Care-accredited institution in accordance with the principles of the National Institutes of Health. All studies were carried out in strict accordance with the recommendations in the Guide for the Care and Use of Laboratory Animals of the National Institutes of Health in BIOQUAL (Rockville, MD). BIOQUAL is fully accredited by AAALAC and through OLAW, Assurance

381 Number A-3086. All physical procedures associated with this work were done under anesthesia to minimize pain and distress in accordance with the recommendations of the Weatherall report, "The use of non-human primates in research.” Teklad 5038 Primate Diet was provided once daily by animal size and weight. The diet was supplemented with fresh fruit and vegetables. Fresh water was given ad libitum. All monkeys were maintained in accordance with the Guide for the Care and Use of Laboratory Animals.

Cynomolgus macaques were on average 8-9 years old and ranged from 2.75 to $8 \mathrm{~kg}$ in body weight.

387 Male and female macaques per group were balanced when availability permitted. Studies were performed 388 unblinded. The RBD-scNP, NTD-scNP and S2P-scNP immunogens were formulated with adjuvants as 389 previously described (Fox et al., 2016) and given intramuscularly in the right and left quadriceps. In the 390 first study, cynomolgus macaques $(n=5)$ were immunized for three times with $100 \mu \mathrm{g}$ of RBD-scNP, 
391 NTD-scNP and S2P-scNP adjuvanted with $5 \mu \mathrm{g}$ of 3M-052 aqueous formulation admixed with $500 \mu \mathrm{g}$ of

392 alum in PBS. In the second study, cynomolgus macaques $(\mathrm{n}=5)$ were were immunized twice with $50 \mu \mathrm{g}$

393 of S-2P mRNA-LNP (encoding the transmembrane spike protein stabilized with K986P and V987P

394 mutations) and boosted once with $100 \mu \mathrm{g}$ of RBD-scNP, NTD-scNP and S2P-scNP adjuvanted with $5 \mu \mathrm{g}$

395 of 3M-052 aqueous formulation admixed with $500 \mu \mathrm{g}$ of alum in PBS. In the third study, cynomolgus

396 macaques were immunized for twice with $100 \mu \mathrm{g}$ of RBD-scNP or recombinant soluble RBD with $5 \mu \mathrm{g}$ of

397 3M-052 aqueous formulation admixed with $500 \mu \mathrm{g}$ of alum in PBS. In the fourth study, macaques were

398 divided into 8 groups ( $\mathrm{n}=5$ per group) as following: 1 ) control group: no immunization; 2 ) immunogen

399 alone group: $100 \mu \mathrm{g}$ of RBD-scNP; 3) RBD-scNP + 3M-052-Alum group: $100 \mu \mathrm{g}$ of RBD-scNP + $5 \mu \mathrm{g}$

400 of 3M-052 in aqueous formulation $+500 \mu \mathrm{g}$ of Alum (i.e. aluminum ion); 4) 3M-052-Alum alone group:

$4015 \mu \mathrm{g}$ of $3 \mathrm{M}-052$ in aqueous formulation $+500 \mu \mathrm{g}$ of Alum; 5) RBD-scNP + Alum group: $100 \mu \mathrm{g}$ of RBD-

$402 \mathrm{scNP}+500 \mu \mathrm{g}$ of Alum; 6) Alum alone group: $500 \mu \mathrm{g}$ of Alum; 7) RBD-scNP + 3M-052-AF group: 100

$403 \mu \mathrm{g}$ of RBD-scNP $+5 \mu \mathrm{g}$ of 3M-052 in aqueous formulation; 8) 3M-052-AF alone group: $5 \mu \mathrm{g}$ of 3M-052

404 in aqueous formulation.

SARS-CoV-2 viral challenge

For SARS-CoV-2 challenge, $10^{5}$ plaque-forming units (PFU) of SARS-CoV-2 virus Isolate USAWA1/2020 ( 10 $0^{6}$ TCID50) were diluted in $4 \mathrm{~mL}$ and were given by $1 \mathrm{~mL}$ intranasally and $3 \mathrm{~mL}$ intratracheally on Day 0. Biospecimens, including nasal swabs, BAL, plasma, and serum samples, were

410 collected before immunization, after every immunization, before challenge, 2 days post-challenge and 4

411 days post-challenge. Animals were necropsied on Day 4 post-challenge, and lungs were collected for

412 histopathology and immunohistochemistry (IHC) analysis.

\section{Recombinant protein production}

415 The coronavirus ectodomain proteins were produced and purified as previously described (Li et al., 416 2021a; Saunders et al., 2021; Wrapp et al., 2020; Zhou et al., 2020). S-2P was stabilized by the 
417 introduction of 2 prolines at amino acid positions 986 and 987. Plasmids encoding SARS-CoV-2 and

418 other coronavirus S-2P (Genscript) were transiently transfected in FreeStyle 293-F cells (Thermo Fisher)

419 using Turbo293 (SpeedBiosystems) or 293Fectin (ThermoFisher). All cells were tested monthly for

420 mycoplasma. The constructs contained an HRV 3C-cleavable C-terminal twinStrepTagII- $8 \times$ His tag. On

421 day 6 , cell-free culture supernatant was generated by centrifugation of the culture and filtering through a

$422 \quad 0.8-\mu \mathrm{m}$ filter. Protein was purified from filtered cell culture supernatants by StrepTactin resin (IBA) and

423 by size-exclusion chromatography using Superdex 200 (RBD and NTD) or Superose 6 (S-2P and ferritin)

424 column (GE Healthcare) in $10 \mathrm{mM}$ Tris $\mathrm{pH}=8,500 \mathrm{mM} \mathrm{NaCl}$. ACE2-Fc was expressed by transient

425 transfection of Freestyle 293-F cells. ACE2-Fc was purified from cell culture supernatant by HiTrap

426 protein A column chromatography and Superdex200 size-exclusion chromatography in $10 \mathrm{mM}$ Tris

$427 \mathrm{pH} 8,150 \mathrm{mM} \mathrm{NaCl}$. SARS-CoV-2 RBD and NTD were produced as previously described (Saunders et

428 al., 2021; Zhou et al., 2020).

RBD-scNP, NTD-scNP, and S2P-scNP were produced by conjugating SARS-CoV-2 RBD to $H$.

430 pylori ferritin nanoparticles using Sortase A as previously described (Saunders et al., 2021). Briefly,

431 SARS-CoV-2 Wuhan strain RBD, NTD or S-2P (with a C-terminal foldon trimerization motif) was

432 expressed with a sortase A donor sequence LPETGG encoded at its C terminus. C-terminal to the sortase

433 A donor sequence was an HRV-3C cleavage site, $8 \times$ His tag and a twin StrepTagII (IBA). The proteins

434 were expressed in Freestyle 293-F cells and purified by StrepTactin affinity chromatography and

435 Superdex 200 or Superose 6 size-exclusion chromatography. Helicobacter pylori ferritin particles were

436 expressed with a pentaglycine sortase $\mathrm{A}$ acceptor sequence encoded at its $\mathrm{N}$ terminus of each subunit. For

437 affinity purification of ferritin particles, $6 \times$ His tags were appended C-terminal to a HRV3C cleavage site.

438 Ferritin particles with a sortase A N-terminal tag were buffer exchanged into $50 \mathrm{mM}$ Tris, $150 \mathrm{mM} \mathrm{NaCl}$,

$4395 \mathrm{mM} \mathrm{CaCl}_{2}, \mathrm{pH}$ 7.5. Then $180 \mu \mathrm{M}$ SARS-CoV-2 RBD was mixed with $120 \mu \mathrm{M}$ of ferritin subunits and

440 incubated with $100 \mu \mathrm{M}$ of sortase A overnight at room temperature. Following incubation, conjugated

441 particles were isolated from free ferritin or free RBD/NTD/S-2P by size-exclusion chromatography using

442 a Superose 6 16/60 column. 


\section{mRNA-LNP vaccine production}

$$
\text { The S-2P mRNA was designed based on the SARS-CoV-2 spike (S) protein sequence (Wuhan-Hu-1) }
$$
and encoded the full-length S with K986P and V987P amino acid substitutions. Production of the mRNA

447 was performed as described earlier (Freyn et al., 2021; Freyn et al., 2020). Briefly, the codon-optimized

448 S-2P gene was synthesized (Genscript) and cloned into an mRNA production plasmid. A T7-driven in

449 vitro transcription reaction (Megascript, Ambion) using linearized plasmid template was performed to

450 generate mRNA with 101 nucleotide long poly(A) tail. Capping of the mRNA was performed in concert

451 with transcription through addition of a trinucleotide cap1 analog, CleanCap (TriLink) and m1 1 -5'-

452 triphosphate (TriLink) was incorporated into the reaction instead of UTP. Cellulose-based purification of

453 S-2P mRNA was performed as described (Baiersdorfer et al., 2019). The S-2P mRNA was then tested on

454 an agarose gel before storing at $-20^{\circ} \mathrm{C}$. The cellulose-purified $\mathrm{m} 1 \Psi$-containing S-2P mRNA was

455 encapsulated in LNPs using a self-assembly process as previously described wherein an ethanolic lipid

456 mixture of ionizable cationic lipid, phosphatidylcholine, cholesterol and polyethylene glycol-lipid was

457 rapidly mixed with an aqueous solution containing mRNA at acidic pH (Maier et al., 2013). The RNA-

458 loaded particles were characterized and subsequently stored at $80^{\circ} \mathrm{C}$ at a concentration of $1 \mathrm{mg} / \mathrm{ml}$.

\section{Antibody Binding ELISA}

For binding ELISA, 384-well ELISA plates were coated with $2 \mu \mathrm{g} / \mathrm{mL}$ of antigens in $0.1 \mathrm{M}$ sodium

462 bicarbonate overnight at $4^{\circ} \mathrm{C}$. Plates were washed with PBS $+0.05 \%$ Tween 20 and blocked with blocked

463 with assay diluent (PBS containing 4\% (w/v) whey protein, 15\% Normal Goat Serum, 0.5\% Tween-20,

464 and $0.05 \%$ Sodium Azide) at room temperature for 1 hour. Plasma or mucosal fluid were serially diluted

465 threefold in superblock starting at a 1:30 dilution. Nasal fluid was started from neat, whereas BAL fluid

466 was concentrated ten-fold. To concentrate BAL, individual BAL aliquots from the same macaque and

467 same time point were pooled in 3-kDa MWCO ultrafiltration tubes (Sartorious, catalog \# VS2091).

468 Pooled BAL was concentrated by centrifugation at 3,500 rpm for $30 \mathrm{~min}$ or until volume was reduced by 
469 a factor of 10 . The pool was then aliquoted and frozen at $-80{ }^{\circ} \mathrm{C}$ until its use in an assay. Serially diluted

470 samples were added and incubated for 1 hour, followed by washing with PBS-0.1\% Tween 20. HRP-

471 conjugated goat anti-human IgG secondary Ab (SouthernBiotech, catalog\# 2040-05) was diluted to

$4721: 10,000$ and incubated at room temperature for 1 hour. These plates were washed four times and

473 developed with tetramethylbenzidine substrate (SureBlue Reserve- KPL). The reaction was stopped with

$4741 \mathrm{M} \mathrm{HCl}$, and optical density at $450 \mathrm{~nm}\left(\mathrm{OD}_{450}\right)$ was determined.

\section{ACE2 and neutralizing antibody blocking assay}

ELISA plates were coated as stated above with $2 \mu \mathrm{g} / \mathrm{mL}$ recombinant ACE-2 protein or neutralizing

478 antibodies, then washed and blocked with 3\% BSA in 1x PBS. While assay plates blocked, plasma or

479 mucosal samples were diluted as stated above, only in $1 \%$ BSA with $0.05 \%$ Tween-20. In a separate

480 dilution plate spike-2P protein was mixed with the antibodies at a final concentration equal to the EC50 at

481 which spike binds to ACE-2 protein. The mixture was incubated at room temperature for 1 hour. Blocked

482 assay plates were then washed and the antibody-spike mixture was added to the assay plates for a period

483 of 1 hour at room temperature. Plates were washed and a polyclonal rabbit serum against the same spike

484 protein (nCoV-1 nCoV-2P.293F) was added for 1 hour, washed and detected with goat anti rabbit-HRP

485 (Abcam catalog \# ab97080) followed by TMB substrate. The extent to which antibodies were able to

486 block the binding spike protein to ACE-2 or neutralizing antibodies was determined by comparing the OD

487 of antibody samples at $450 \mathrm{~nm}$ to the $\mathrm{OD}$ of samples containing spike protein only with no antibody. The

488 following formula was used to calculate percent blocking: blocking $\%=(100-(\mathrm{OD}$ sample/OD of spike

489 only)*100).

\section{Pseudotyped SARS-CoV-2 neutralization assay}

492 Neutralization of SARS-CoV-2 Spike-pseudotyped virus was performed by adopting an infection 493 assay described previously (Korber et al., 2020) with lentiviral vectors and infection in 293T/ACE2.MF

494 (the cell line was kindly provided by Drs. Mike Farzan and Huihui Mu at Scripps). Cells were maintained 
495 in DMEM containing 10\% FBS and $50 \mu \mathrm{g} / \mathrm{ml}$ gentamicin. An expression plasmid encoding codon-

496 optimized full-length spike of the Wuhan-1 strain (VRC7480), was provided by Drs. Barney Graham and

497 Kizzmekia Corbett at the Vaccine Research Center, National Institutes of Health (USA). Mutations were

498 introduced into VRC7480 either by site-directed mutagenesis using the QuikChange Lightning Site-

499 Directed Mutagenesis Kit from Agilent Technologies (Catalog \# 210518), or were created by spike gene

500 synthesized by GenScript using the spike sequence in VRC7480 as template. All mutations were

501 confirmed by full-length spike gene sequencing by Sanger Sequencing, using Sequencher and SnapGene

502 for sequence analyses. D614G spike mutation: D614G; Alpha (B.1.1.7) spike mutations: $\Delta 69-70, \Delta 144$,

503 N501Y, A570D, D614G, P681H, T716I, S982A, D1118H; Beta (B.1.351) spike mutations: L18F, D80A,

504 D215G, _242-244, R246I, K417N, E484K, N501Y, D614G, A701V; Delta (B.1.617 AY.3) spike

505 mutations: T19R, G142D, $\Delta 156-157$, R158G, L452R, T478K, D614G, P681R, D950N; Epsilon (B.1.429)

506 spike mutations: S13I, W152C, L452R, D614G; Omicron (B.1.1.529) spike mutations: A67V, $\Delta 69-70$,

507 T95I, G142D, $\Delta 143-145, \Delta 211$, L212I, +214EPE, G339D, S371L, S373P, S375F, K417N, N440K,

508 G446S, S477N, T478K, E484A, Q493R, G496S, Q498R, N501Y, Y505H, T547K, D614G, H655Y,

509 N679K, P681H, N764K, D796Y, N856K, Q954H, N969K, L981F. Pseudovirions were produced in HEK

510 293T/17 cells (ATCC cat. no. CRL-11268) by transfection using Fugene 6 (Promega, Catalog \#E2692).

511 Pseudovirions for 293T/ACE2 infection were produced by co-transfection with a lentiviral backbone

512 (pCMV $\Delta$ R8.2) and firefly luciferase reporter gene (pHR' CMV Luc) (Naldini et al., 1996). Culture

513 supernatants from transfections were clarified of cells by low-speed centrifugation and filtration $(0.45 \mu \mathrm{m}$

514 filter) and stored in $1 \mathrm{ml}$ aliquots at $-80^{\circ} \mathrm{C}$. A pre-titrated dose of virus was incubated with 8 serial 3-fold

515 or 5-fold dilutions of mAbs in duplicate in a total volume of $150 \mu \mathrm{l}$ for $1 \mathrm{hr}$ at $37^{\circ} \mathrm{C}$ in 96 -well flat-

516 bottom poly-L-lysine-coated culture plates (Corning Biocoat). Cells were suspended using TrypLE

517 express enzyme solution (Thermo Fisher Scientific) and immediately added to all wells (10,000 cells in

$518100 \mu \mathrm{L}$ of growth medium per well). One set of 8 control wells received cells + virus (virus control) and

519 another set of 8 wells received cells only (background control). After 66-72 hrs of incubation, medium 
520 was removed by gentle aspiration and $30 \mu \mathrm{L}$ of Promega $1 \mathrm{x}$ lysis buffer was added to all wells. After a

521 10-minute incubation at room temperature, $100 \mu 1$ of Bright-Glo luciferase reagent was added to all wells.

522 After 1-2 minutes, $110 \mu 1$ of the cell lysate was transferred to a black/white plate (Perkin-Elmer).

523 Luminescence was measured using a PerkinElmer Life Sciences, Model Victor2 luminometer.

524 Neutralization titers are the mAb concentration (IC50/IC80) at which relative luminescence units (RLU)

525 were reduced by $50 \%$ and $80 \%$ compared to virus control wells after subtraction of background RLUs.

526 Negative neutralization values are indicative of infection-enhancement. Maximum percent inhibition

527 (MPI) is the reduction in RLU at the highest $\mathrm{mAb}$ concentration tested.

528 Another protocol was used to test plasma neutralization against pseudoviruses of SARS-CoV-2 WA-

5291 strain and variants. Human codon-optimized cDNA encoding SARS-CoV-2 spike glycoproteins of

530 various strains were synthesized by GenScript and cloned into eukaryotic cell expression vector pcDNA

5313.1 between the BamHI and XhoI sites. Pseudovirions were produced by co-transfection of Lenti-X 293T

532 cells with psPAX2(gag/pol), pTrip-luc lentiviral vector and pcDNA 3.1 SARS-CoV-2-spike-deltaC19,

533 using Lipofectamine 3000. The supernatants were collected at $48 \mathrm{~h}$ after transfection and filtered through

$534 \quad 0.45-\mu \mathrm{m}$ membranes and titrated using HEK293T cells that express ACE2 and TMPRSS2 protein (293T-

535 ACE2-TMPRSS2 cells). For the neutralization assay, $50 \mu \mathrm{l}$ of SARS-CoV-2 spike pseudovirions were

536 pre-incubated with an equal volume of medium containing serum at varying dilutions at room temperature

537 for $1 \mathrm{~h}$, then virus-antibody mixtures were added to 293T-ACE2-TMPRSS2 cells in a 96-well plate. After

538 a 3-h incubation, the inoculum was replaced with fresh medium. Cells were lysed $24 \mathrm{~h}$ later, and

539 luciferase activity was measured using luciferin. Controls included cell-only control, virus without any

540 antibody control and positive control sera. Neutralization titres are the serum dilution (ID50 or ID80) at

541 which relative luminescence units (RLU) were reduced by $50 \%$ or $80 \%$, respectively, compared to virus

542 control wells after subtraction of background RLUs.

\section{Live SARS-CoV-2 neutralization assays}



neutralization (MN) assays were adapted from a previous study (Berry et al., 2004). In short, sera or purified Abs are diluted two-fold and incubated with 100 TCID50 virus for 1 hour. These dilutions are used as the input material for a TCID50. Each batch of MN includes a known neutralizing control Ab

550 (Clone D001; SINO, CAT\# 40150-D001). Data are reported as the concentration at which 50\% of input

551 virus is neutralized. A known neutralizing control antibody is included in each batch run (Clone D001;

552 SINO, CAT\# 40150-D001). GraphPad Prism was used to determine $\mathrm{ID}_{50}$ values.

\section{Spike protein-expressing cell antibody binding assay}

The cell antibody binding assay was performed as previously described (Pino et al., 2021). Briefly, target cells were derived by transfection with plasmids designed to express the SARS-CoV-2 D614 Spike protein with a c-terminus flag tag (kindly provided by Dr. Farzan, Addgene plasmid no. 156420 (Zhang et al., 2020)). Cells not transfected with any plasmid (mock transfected) were used as a negative control condition. After resuspension, washing and counting, $1 \times 10^{5}$ Spike-transfected target cells were dispensed into 96-well V-bottom plates and incubated with six serial dilutions of macaque plasma starting at 1:50 dilution. Mock transfected cells were used as a negative infection control. After 30 minutes incubation at $37^{\circ} \mathrm{C}$, cells are washed twice with $250 \mu \mathrm{L} /$ well of PBS, stained with vital dye (Live/Dead Far Red Dead

563 Cell Stain, Invitrogen) to exclude nonviable cells from subsequent analysis, washed with Wash Buffer

564 (1\%FBS-PBS; WB), permeabilized with CytoFix/CytoPerm (BD Biosciences), and stained with 1.25

$565 \mu \mathrm{g} / \mathrm{mL}$ anti-human IgG Fc-PE/Cy7 (Clone HP6017; Biolegend) and $5 \mu \mathrm{g} / \mathrm{mL}$ anti-flag-FITC (clone M2;

566 Sigma Aldrich) in the dark for 20 minutes at room temperature. After three washes with Perm Wash (BD

567 Biosciences), the cells were resuspended in $125 \mu \mathrm{L}$ PBS-1\% paraformaldehyde. Samples were acquired

568 within $24 \mathrm{~h}$ using a BD Fortessa cytometer and a High Throughput Sampler (HTS, BD Biosciences). Data

569 analysis was performed using FlowJo 10 software (BD Biosciences). A minimum of 50,000 total events

570 were acquired for each analysis. Gates were set to include singlet, live, flag + and IgG + events. All final 
571 data represent specific binding, determined by subtraction of non-specific binding observed in assays

572 performed with mock-transfected cells.

\section{Antibody-dependent NK cell degranulation assay}

575 Cell-surface expression of CD107a was used as a marker for NK cell degranulation, a prerequisite 576 process for ADCC (Ferrari et al., 2011), was performed as previously described (Pino et al., 2021).

577 Briefly, target cells were either Vero E6 cells after a 2 day-infection with SARS-CoV-2 USA-WA1/2020

578 or 293 T cells 2-days post transfection with a SARS-CoV-2 S protein (D614) expression plasmid. NK

579 cells were purified from peripheral blood of a healthy human volunteer by negative selection (Miltenyi

580 Biotech), and were incubated with target cells at a 1:1 ratio in the presence of diluted plasma or

581 monoclonal antibodies, Brefeldin A (GolgiPlug, $1 \mu \mathrm{l} / \mathrm{ml}$, BD Biosciences), monensin (GolgiStop,

$5824 \mu \mathrm{l} / 6 \mathrm{~mL}$, BD Biosciences), and anti-CD107a-FITC (BD Biosciences, clone H4A3) in 96-well flat bottom

583 plates for 6 hours at $37^{\circ} \mathrm{C}$ in a humidified $5 \% \mathrm{CO} 2$ incubator. NK cells were then recovered and stained

584 for viability prior to staining with CD56-PECy7 (BD Biosciences, clone NCAM16.2), CD16-PacBlue

585 (BD Biosciences, clone 3G8), and CD69-BV785 (Biolegend, Clone FN50). Flow cytometry data analysis

586 was performed using FlowJo software (v10.8.0). Data is reported as the \% of CD107A+ live NK cells

587 (gates included singlets, lymphocytes, aqua blue-, CD56+ and/or CD16+, CD107A+). All final data

588 represent specific activity, determined by subtraction of non-specific activity observed in assays

589 performed with mock-infected cells and in absence of antibodies.

\section{Viral RNA Extraction and Subgenomic mRNA quantification}

592 SARS-CoV-2 E gene and N gene subgenomic mRNA (sgRNA) was measured by a one-step RT-

593 qPCR adapted from previously described methods (Wolfel et al., 2020; Yu et al., 2020). To generate

594 standard curves, a SARS-CoV-2 E gene sgRNA sequence, including the 5'UTR leader sequence,

595 transcriptional regulatory sequence (TRS), and the first 228 bp of E gene, was cloned into a pcDNA3.1

596 plasmid. For generating SARS-CoV-2 N gene sgRNA, the E gene was replaced with the first 227 bp of N 
gene. The recombinant pcDNA3.1 plasmid was linearized, transcribed using MEGAscript T7

Transcription Kit (ThermoFisher, catalog \# AM1334), and purified with MEGAclear Transcription

Clean-Up Kit (ThermoFisher, catalog \# AM1908). The purified RNA products were quantified on

600 Nanodrop, serial diluted, and aliquoted as E sgRNA or N sgRNA standards.

601 A QIAsymphony SP (Qiagen, Hilden, Germany) automated sample preparation platform along with a

602 virus/pathogen DSP midi kit. RNA extracted from animal samples or standards were then measured in

603 Taqman custom gene expression assays (ThermoFisher). For these assays we used TaqMan Fast Virus 1-

604 Step Master Mix (ThermoFisher, catalog \# 4444432) and custom primers/probes targeting the E gene

605 sgRNA (forward primer: 5' CGA TCT CTT GTA GAT CTG TTC TCE 3'; reverse primer: 5' ATA TTG

606 CAG CAG TAC GCA CAC A 3'; probe: 5' FAM-ACA CTA GCC ATC CTT ACT GCG CTT CG-

607 BHQ1 3') or the N gene sgRNA (forward primer: 5' CGA TCT CTT GTA GAT CTG TTC TC 3';

608 reverse primer: 5' GGT GAA CCA AGA CGC AGT AT 3'; probe: 5' FAM-TAA CCA GAA TGG AGA

609 ACG CAG TGG G-BHQ1 3'). RT-qPCR reactions were carried out on CFX384 Touch Real-Time PCR

610 System (Bio-Rad) using a program below: reverse transcription at $50^{\circ} \mathrm{C}$ for 5 minutes, initial denaturation

611 at $95^{\circ} \mathrm{C}$ for 20 seconds, then 40 cycles of denaturation-annealing-extension at $95^{\circ} \mathrm{C}$ for 15 seconds and

$61260^{\circ} \mathrm{C}$ for 30 seconds. Standard curves were used to calculate E or N sgRNA in copies per ml; the limit of

613 detections (LOD) for both $\mathrm{E}$ and $\mathrm{N}$ sgRNA assays were 12.5 copies per reaction or 150 copies per $\mathrm{mL}$ of

$614 \mathrm{BAL} /$ nasal swab.

\section{Histopathology}

617 Lung specimen from nonhuman primates were fixed in 10\% neutral buffered formalin, processed,

618 and blocked in paraffin for histological analysis. All samples were sectioned at $5 \mu \mathrm{m}$ and stained with

619 hematoxylin-eosin (H\&E) for routine histopathology. Sections were examined under light microscopy

620 using an Olympus BX51 microscope and photographs were taken using an Olympus DP73 camera.

621 Samples were scored by a board-certified veterinary pathologist in a blinded manner. The representative 
622 images are to characterize the types and arrangement of inflammatory cells, while the scores show the

623 relative severity of the tissue section.

\section{Immunohistochemistry (IHC)}

626 Staining for SARS-CoV-2 antigen was achieved on the Bond RX automated system with the

627 Polymer Define Detection System (Leica) used per manufacturer's protocol. Tissue sections were

628 dewaxed with Bond Dewaxing Solution (Leica) at $72^{\circ} \mathrm{C}$ for 30 min then subsequently rehydrated with

629 graded alcohol washes and 1x Immuno Wash (StatLab). Heat-induced epitope retrieval (HIER) was

630 performed using Epitope Retrieval Solution 1 (Leica), heated to $100^{\circ} \mathrm{C}$ for 20 minutes. A peroxide block

631 (Leica) was applied for $5 \mathrm{~min}$ to quench endogenous peroxidase activity prior to applying the SARS-

632 CoV-2 antibody (1:2000, GeneTex, GTX135357). Antibodies were diluted in Background Reducing

633 Antibody Diluent (Agilent). The tissue was subsequently incubated with an anti-rabbit HRP polymer

634 (Leica) and colorized with 3,3'-Diaminobenzidine (DAB) chromogen for $10 \mathrm{~min}$. Slides were

635 counterstained with hematoxylin.

\section{Negative-stain electron microscopy}

Samples diluted to $200 \mu \mathrm{g} / \mathrm{ml}$ with $5 \mathrm{~g} / \mathrm{dl}$ Glycerol in HBS (20 mM HEPES, $150 \mathrm{mM} \mathrm{NaCl} \mathrm{pH}$

7.4) buffer containing $8 \mathrm{mM}$ glutaraldehyde. After $5 \mathrm{~min}$ incubation, glutaraldehyde was quenched by adding sufficient $1 \mathrm{M}$ Tris stock, $\mathrm{pH} 7.4$, to give $75 \mathrm{mM}$ final Tris concentration and incubated for $5 \mathrm{~min}$.

641 Quenched sample was applied to a glow-discharged carbon-coated EM grid for 10-12 second, then

642 blotted, and stained with $2 \mathrm{~g} / \mathrm{dL}$ uranyl formate for $1 \mathrm{~min}$, blotted and air-dried. Grids were examined on a

643 Philips EM420 electron microscope operating at $120 \mathrm{kV}$ and nominal magnification of 82,000x, and

644 images were collected on a 4 Mpix CCD camera at $4 \AA$ pixel. Images were analyzed by $2 \mathrm{D}$ class averages

645 using standard protocols with Relion 3.0 (Zivanov et al., 2018). The Relion reference needs to be added to

646 the references. Also let us know which lot\# you are shown in this manuscript so we can double check

647 whether images are collected with old or new camera. Since the magnification and $\AA$ /pixel is different. 


\section{Mouse immunization and challenge}

Eleven-month-old female BALB/c mice were purchased from Envigo (\#047) and were used for the SARS-CoV, SARS-CoV-2 WA-1, SARS-CoV-2 B.1.351, and RsSHC014-CoV protection experiments.

652 The study was carried out in accordance with the recommendations for care and use of animals by the

653 Office of Laboratory Animal Welfare (OLAW), National Institutes of Health and the Institutional Animal

654 Care and Use Committee (IACUC) of University of North Carolina (UNC permit no. A-3410-01).

655 Animals were housed in groups of five and fed standard chow diets. Virus inoculations were performed

656 under anesthesia and all efforts were made to minimize animal suffering. Mice were intramuscularly

657 immunized with RBD-scNP formulated with 3M-052-AF + Alum or GLA-SE. For the SARS-CoV-2

658 WA-1 and RsSHC014 study, mice were immunized on week 0 and 2, and challenged on week 7. For the

659 SARS-CoV-2 B.1.351 and SARS-CoV-1 study, mice were immunized on week 0 and 4, and challenged

660 on week 6 . All mice were anesthetized and infected intranasally with $1 \times 10^{4} \mathrm{PFU} / \mathrm{ml}$ of SARS-CoV

661 MA15, $1 \times 10^{4} \mathrm{PFU} / \mathrm{ml}$ of SARS-CoV-2 WA1- MA10 or B.1.351-MA10, $1 \times 10^{4} \mathrm{PFU} / \mathrm{ml}$ RsSHC014,

662 which have been described previously (Leist et al., 2020; Martinez et al., 2021a; Martinez et al., 2021b;

663 Menachery et al., 2015; Roberts et al., 2007). Mice were weighted daily and monitored for signs of

664 clinical disease, and selected groups were subjected to daily whole-body plethysmography. For all mouse

665 studies, groups of $n=10$ mice were included per arm of the study. Lung viral titers and weight loss were

666 measured from individual mice per group.

\section{Biocontainment and biosafety}

Studies were approved by the UNC Institutional Biosafety Committee approved by animal and

670 experimental protocols in the Baric laboratory. All work described here was performed with approved

671 standard operating procedures for SARS-CoV-2 in a biosafety level 3 (BSL-3) facility conforming to

672 requirements recommended in the Microbiological and Biomedical Laboratories, by the U.S. Department 
of Health and Human Service, the U.S. Public Health Service, and the U.S. Center for Disease Control

674 and Prevention (CDC), and the National Institutes of Health (NIH).

\section{Statistics Analysis}

Data were plotted using Prism GraphPad 8.0. Wilcoxon rank sum exact test was performed to

678 compare differences between groups with $p$-value $<0.05$ considered significant using SAS 9.4 (SAS

679 Institute, Cary, NC). The Benjamini-Hochberg correction (Benjamini and Hochberg, 1995) was used to 680 adjust the $p$-values for multiple comparisons.

DATA AVAILABILITY

The data that support the findings of this study are available from the corresponding authors upon reasonable request.

\section{AUTHOR CONTRIBUTIONS}

D.L. performed RNA assays, analyzed the data and wrote the manuscript; D.R.M., A.S., and R.S.B. performed or supervised the mouse challenge studies; L.L.S., M.B., W.E., A.N. performed monkey studies and managed monkey samples; H.C., E.L., A.B. performed protein production; M.B., R.P. carried

691 out ELISA assays; T.H.O., G.D.S., D.C.M., A.E., H.G. and S.K. carried out neutralization assays; D.L., 692 C.T.M., T.N.D., M.G., D.C.D. designed or performed subgenomic RNA assays; K.W.B., M.M., B.M.N., 693 I.N.M. performed histopathology analysis; D.M., G.F., D.W.C., and S.S. performed staining and ADCC

694 assays; K.M. and R.J.E. performed negative staining EM; R.W.R. and Y.W. performed statistical 695 analyses; M.A.T. selected and provided adjuvant; C.B.F. formulated 3M-052 in alum; N.P. and D.W.

696 provided the mRNA-LNP vaccine; M.A.T. selected and provided adjuvant; C.B.F. formulated 3M-052;

697 M.G.L., H.A. and R.S. evaluated and supervised monkey studies; K.O.S. designed the antigens, 
698 supervised the protein production, and edited the manuscript; B.F.H. designed and managed the study,

699 reviewed all data and wrote and edited the manuscript. All authors edited and approved the manuscript.

\section{COMPETING FINANCIAL INTERESTS}

B.F.H. and K.O.S. have filed US patents regarding the nanoparticle vaccine, M.A.T. and the 3M company have US patents filed on 3M-052, and C.B.F. and IDRI have filed a patent on the formulation of 3M-052-AF and 3M-052-AF + Alum. The 3M company had no role in the execution of the study, data collection or data interpretation. D.W. is named on US patents that describe the use of nucleosidemodified mRNA as a platform to deliver therapeutic proteins. D.W. and N.P. are also named on a US

707 patent describing the use of nucleoside-modified mRNA in lipid nanoparticles as a vaccine platform. All

708 other authors declare no competing interests.

\section{ACKNOWLEDGEMENTS}

711 We thank Margaret Deyton, Victoria Gee-Lai, Aja Sanzone, Nolan Jamieson, Lena Smith, Nicole De

712 Naeyer and Conor Anderson for technical assistance. We thank Elizabeth Donahue, Cynthia Nagle and

713 Kelly Soderberg for program management. We thank John Harrison, Alex Granados, Adrienne Goode,

714 Anthony Cook, Alan Dodson, Katelyn Steingrebe, Bridget Bart, Laurent Pessaint, Alex VanRy, Daniel

715 Valentin, Amanda Strasbaugh, and Mehtap Cabus for assistance with macaque studies. This work was

716 supported by funds from the State of North Carolina with funds from the federal CARES Act; NIH,

717 NIAID, DAIDS grant AI142596 (B.F.H.), AI158571 (B.F.H.), UC6-AI058607, G20-AI167200 (G.D.S.);

718 the Ting Tsung \& Wei Fong Chao Foundation (B.F.H.); Hanna H Gray Fellowship from the Howard

719 Hughes Medical Institute and a Postdoctoral Enrichment Award from the Burroughs Wellcome Fund 720 (D.R.M.).

\section{REFERENCES}


723 Arvin, A.M., Fink, K., Schmid, M.A., Cathcart, A., Spreafico, R., Havenar-Daughton, C., Lanzavecchia,

724 A., Corti, D., and Virgin, H.W. (2020). A perspective on potential antibody-dependent enhancement of

725 SARS-CoV-2. Nature 584, 353-363. 10.1038/s41586-020-2538-8.

726 Baden, L.R., El Sahly, H.M., Essink, B., Kotloff, K., Frey, S., Novak, R., Diemert, D., Spector, S.A.,

727 Rouphael, N., Creech, C.B., et al. (2021). Efficacy and Safety of the mRNA-1273 SARS-CoV-2 Vaccine.

728 N Engl J Med 384, 403-416. 10.1056/NEJMoa2035389.

729 Baiersdorfer, M., Boros, G., Muramatsu, H., Mahiny, A., Vlatkovic, I., Sahin, U., and Kariko, K. (2019).

730 A Facile Method for the Removal of dsRNA Contaminant from In Vitro-Transcribed mRNA. Mol Ther

731 Nucleic Acids 15, 26-35. 10.1016/j.omtn.2019.02.018.

732 Benjamini, Y., and Hochberg, Y. (1995). Controlling the False Discovery Rate: A Practical and Powerful

733 Approach to Multiple Testing. Journal of the Royal Statistical Society. Series B (Methodological) 57,

$734 \quad 289-300$.

735 Berry, J.D., Jones, S., Drebot, M.A., Andonov, A., Sabara, M., Yuan, X.Y., Weingartl, H., Fernando, L.,

736 Marszal, P., Gren, J., et al. (2004). Development and characterisation of neutralising monoclonal antibody

737 to the SARS-coronavirus. J Virol Methods 120, 87-96. 10.1016/j.jviromet.2004.04.009.

738 Cameroni, E., Bowen, J.E., Rosen, L.E., Saliba, C., Zepeda, S.K., Culap, K., Pinto, D., VanBlargan, L.A.,

739 De Marco, A., di Iulio, J., et al. (2021). Broadly neutralizing antibodies overcome SARS-CoV-2 Omicron

740 antigenic shift. Nature. 10.1038/s41586-021-04386-2.

741 Cerutti, G., Guo, Y., Zhou, T., Gorman, J., Lee, M., Rapp, M., Reddem, E.R., Yu, J., Bahna, F., Bimela,

742 J., et al. (2021). Potent SARS-CoV-2 neutralizing antibodies directed against spike N-terminal domain

743 target a single supersite. Cell Host Microbe. 10.1016/j.chom.2021.03.005.

744 Chaudhary, N., Weissman, D., and Whitehead, K.A. (2021). mRNA vaccines for infectious diseases:

745 principles, delivery and clinical translation. Nat Rev Drug Discov 20, 817-838. 10.1038/s41573-021-

$746 \quad 00283-5$. 
Chi, X., Yan, R., Zhang, J., Zhang, G., Zhang, Y., Hao, M., Zhang, Z., Fan, P., Dong, Y., Yang, Y., et al. (2020). A potent neutralizing human antibody reveals the N-terminal domain of the Spike protein of SARS-CoV-2 as a site of vulnerability. 10.1101/2020.05.08.083964.

750 Coffman, R.L., Sher, A., and Seder, R.A. (2010). Vaccine adjuvants: putting innate immunity to work.

751 Immunity 33, 492-503. 10.1016/j.immuni.2010.10.002.

752 Dai, L., Zheng, T., Xu, K., Han, Y., Xu, L., Huang, E., An, Y., Cheng, Y., Li, S., Liu, M., et al. (2020). A

753 Universal Design of Betacoronavirus Vaccines against COVID-19, MERS, and SARS. Cell 182, 722-733

754 e711. 10.1016/j.cell.2020.06.035.

755 Fox, C.B., Kramer, R.M., Barnes, V.L., Dowling, Q.M., and Vedvick, T.S. (2013). Working together:

756 interactions between vaccine antigens and adjuvants. Ther Adv Vaccines 1, 7-20.

$757 \quad 10.1177 / 2051013613480144$

758 Fox, C.B., Orr, M.T., Van Hoeven, N., Parker, S.C., Mikasa, T.J., Phan, T., Beebe, E.A., Nana, G.I.,

759 Joshi, S.W., Tomai, M.A., et al. (2016). Adsorption of a synthetic TLR7/8 ligand to aluminum

760 oxyhydroxide for enhanced vaccine adjuvant activity: A formulation approach. J Control Release 244, 98-

$761 \quad$ 107. 10.1016/j.jconrel.2016.11.011.

762 Francica, J.R., Flynn, B.J., Foulds, K.E., Noe, A.T., Werner, A.P., Moore, I.N., Gagne, M., Johnston,

763 T.S., Tucker, C., Davis, R.L., et al. (2021). Protective antibodies elicited by SARS-CoV-2 spike protein

764 vaccination are boosted in the lung after challenge in nonhuman primates. Sci Transl Med 13.

$76510.1126 /$ scitranslmed.abi4547.

766 Freyn, A.W., Pine, M., Rosado, V.C., Benz, M., Muramatsu, H., Beattie, M., Tam, Y.K., Krammer, F., 767 Palese, P., Nachbagauer, R., et al. (2021). Antigen modifications improve nucleoside-modified mRNA768 based influenza virus vaccines in mice. Mol Ther Methods Clin Dev 22, 84-95.

$769 \quad 10.1016 /$ j.omtm.2021.06.003.

770 Freyn, A.W., Ramos da Silva, J., Rosado, V.C., Bliss, C.M., Pine, M., Mui, B.L., Tam, Y.K., Madden,

771 T.D., de Souza Ferreira, L.C., Weissman, D., et al. (2020). A Multi-Targeting, Nucleoside-Modified 
772 mRNA Influenza Virus Vaccine Provides Broad Protection in Mice. Mol Ther 28, 1569-1584.

773 10.1016/j.ymthe.2020.04.018.

774 Gagne, M., Moliva, J.I., Foulds, K.E., Andrew, S.F., Flynn, B.J., Werner, A.P., Wagner, D.A., Teng, I.-

775 T., Lin, B.C., Moore, C., et al. (2022). mRNA-1273 or mRNA-Omicron boost in vaccinated macaques

776 elicits comparable B cell expansion, neutralizing antibodies and protection against Omicron. bioRxiv,

$777 \quad 2022.2002 .2003 .479037 .10 .1101 / 2022.02 .03 .479037$.

778 Hastie, K.M., Li, H., Bedinger, D., Schendel, S.L., Dennison, S.M., Li, K., Rayaprolu, V., Yu, X., Mann,

779 C., Zandonatti, M., et al. (2021). Defining variant-resistant epitopes targeted by SARS-CoV-2 antibodies:

780 A global consortium study. Science 374, 472-478. 10.1126/science.abh2315.

781 Haynes, B.F., Corey, L., Fernandes, P., Gilbert, P.B., Hotez, P.J., Rao, S., Santos, M.R., Schuitemaker,

782 H., Watson, M., and Arvin, A. (2020). Prospects for a safe COVID-19 vaccine. Sci Transl Med 12.

783 10.1126/scitranslmed.abe0948.

784 HogenEsch, H., O'Hagan, D.T., and Fox, C.B. (2018). Optimizing the utilization of aluminum adjuvants

785 in vaccines: you might just get what you want. NPJ Vaccines 3, 51. 10.1038/s41541-018-0089-x.

786 Kasturi, S.P., Rasheed, M.A.U., Havenar-Daughton, C., Pham, M., Legere, T., Sher, Z.J., Kovalenkov,

787 Y., Gumber, S., Huang, J.Y., Gottardo, R., et al. (2020). 3M-052, a synthetic TLR-7/8 agonist, induces

788 durable HIV-1 envelope-specific plasma cells and humoral immunity in nonhuman primates. Sci

789 Immunol 5. 10.1126/sciimmunol.abb1025.

790 Korber, B., Fischer, W.M., Gnanakaran, S., Yoon, H., Theiler, J., Abfalterer, W., Hengartner, N., Giorgi,

791 E.E., Bhattacharya, T., Foley, B., et al. (2020). Tracking Changes in SARS-CoV-2 Spike: Evidence that

792 D614G Increases Infectivity of the COVID-19 Virus. Cell 182, 812-827 e819.

793 10.1016/j.cell.2020.06.043.

794 Leist, S.R., Dinnon, K.H., 3rd, Schäfer, A., Tse, L.V., Okuda, K., Hou, Y.J., West, A., Edwards, C.E.,

795 Sanders, W., Fritch, E.J., et al. (2020). A Mouse-Adapted SARS-CoV-2 Induces Acute Lung Injury and

796 Mortality in Standard Laboratory Mice. Cell 183, 1070-1085.e1012. 10.1016/j.cell.2020.09.050. 
797 Levin, E.G., Lustig, Y., Cohen, C., Fluss, R., Indenbaum, V., Amit, S., Doolman, R., Asraf, K.,

798 Mendelson, E., Ziv, A., et al. (2021). Waning Immune Humoral Response to BNT162b2 Covid-19

799 Vaccine over 6 Months. N Engl J Med. 10.1056/NEJMoa2114583.

800 Li, D., Edwards, R.J., Manne, K., Martinez, D.R., Schafer, A., Alam, S.M., Wiehe, K., Lu, X., Parks, R.,

801 Sutherland, L.L., et al. (2021a). In vitro and in vivo functions of SARS-CoV-2 infection-enhancing and

802 neutralizing antibodies. Cell 184, 4203-4219 e4232. 10.1016/j.cell.2021.06.021.

803 Li, D., Sempowski, G.D., Saunders, K.O., Acharya, P., and Haynes, B.F. (2021b). SARS-CoV-2

804 Neutralizing Antibodies for COVID-19 Prevention and Treatment. Annu Rev Med. 10.1146/annurev-

805 med-042420-113838.

806 Maier, M.A., Jayaraman, M., Matsuda, S., Liu, J., Barros, S., Querbes, W., Tam, Y.K., Ansell, S.M.,

807 Kumar, V., Qin, J., et al. (2013). Biodegradable lipids enabling rapidly eliminated lipid nanoparticles for

808 systemic delivery of RNAi therapeutics. Mol Ther 21, 1570-1578. 10.1038/mt.2013.124.

809 Marrack, P., McKee, A.S., and Munks, M.W. (2009). Towards an understanding of the adjuvant action of

810 aluminium. Nat Rev Immunol 9, 287-293. 10.1038/nri2510.

811 Martinez, D.R., Schafer, A., Gobeil, S., Li, D., De la Cruz, G., Parks, R., Lu, X., Barr, M., Stalls, V.,

812 Janowska, K., et al. (2021a). A broadly cross-reactive antibody neutralizes and protects against

813 sarbecovirus challenge in mice. Sci Transl Med, eabj7125. 10.1126/scitranslmed.abj7125.

814 Martinez, D.R., Schafer, A., Leist, S.R., De la Cruz, G., West, A., Atochina-Vasserman, E.N.,

815 Lindesmith, L.C., Pardi, N., Parks, R., Barr, M., et al. (2021b). Chimeric spike mRNA vaccines protect

816 against Sarbecovirus challenge in mice. Science 373, 991-998. 10.1126/science.abi4506.

817 McCallum, M., De Marco, A., Lempp, F.A., Tortorici, M.A., Pinto, D., Walls, A.C., Beltramello, M.,

818 Chen, A., Liu, Z., Zatta, F., et al. (2021). N-terminal domain antigenic mapping reveals a site of

819 vulnerability for SARS-CoV-2. Cell. 10.1016/j.cell.2021.03.028.

820 Menachery, V.D., Yount, B.L., Jr., Debbink, K., Agnihothram, S., Gralinski, L.E., Plante, J.A., Graham,

821 R.L., Scobey, T., Ge, X.Y., Donaldson, E.F., et al. (2015). A SARS-like cluster of circulating bat

822 coronaviruses shows potential for human emergence. Nat Med 21, 1508-1513. 10.1038/nm.3985. 
823 Naldini, L., Blomer, U., Gage, F.H., Trono, D., and Verma, I.M. (1996). Efficient transfer, integration,

824 and sustained long-term expression of the transgene in adult rat brains injected with a lentiviral vector.

825 Proc Natl Acad Sci U S A 93, 11382-11388. 10.1073/pnas.93.21.11382.

826 Pardi, N., Hogan, M.J., Porter, F.W., and Weissman, D. (2018). mRNA vaccines - a new era in

827 vaccinology. Nat Rev Drug Discov 17, 261-279. 10.1038/nrd.2017.243.

828 Pardi, N., Hogan, M.J., and Weissman, D. (2020). Recent advances in mRNA vaccine technology. Curr

829 Opin Immunol 65, 14-20. 10.1016/j.coi.2020.01.008.

830 Pino, M., Abid, T., Pereira Ribeiro, S., Edara, V.V., Floyd, K., Smith, J.C., Latif, M.B., Pacheco-Sanchez,

831 G., Dutta, D., Wang, S., et al. (2021). A yeast expressed RBD-based SARS-CoV-2 vaccine formulated

832 with 3M-052-alum adjuvant promotes protective efficacy in non-human primates. Sci Immunol 6.

833 10.1126/sciimmunol.abh3634.

834 Pinto, D., Park, Y.J., Beltramello, M., Walls, A.C., Tortorici, M.A., Bianchi, S., Jaconi, S., Culap, K.,

835 Zatta, F., De Marco, A., et al. (2020). Cross-neutralization of SARS-CoV-2 by a human monoclonal

836 SARS-CoV antibody. Nature 583, 290-295. 10.1038/s41586-020-2349-y.

837 Polack, F.P., Thomas, S.J., Kitchin, N., Absalon, J., Gurtman, A., Lockhart, S., Perez, J.L., Perez Marc,

838 G., Moreira, E.D., Zerbini, C., et al. (2020). Safety and Efficacy of the BNT162b2 mRNA Covid-19

839 Vaccine. N Engl J Med 383, 2603-2615. 10.1056/NEJMoa2034577.

840 Roberts, A., Deming, D., Paddock, C.D., Cheng, A., Yount, B., Vogel, L., Herman, B.D., Sheahan, T.,

841 Heise, M., Genrich, G.L., et al. (2007). A mouse-adapted SARS-coronavirus causes disease and mortality

842 in BALB/c mice. PLoS Pathog 3, e5. 10.1371/journal.ppat.0030005.

843 Routhu, N.K., Cheedarla, N., Bollimpelli, V.S., Gangadhara, S., Edara, V.V., Lai, L., Sahoo, A.,

844 Shiferaw, A., Styles, T.M., Floyd, K., et al. (2021). SARS-CoV-2 RBD trimer protein adjuvanted with

845 Alum-3M-052 protects from SARS-CoV-2 infection and immune pathology in the lung. Nat Commun 12,

$846 \quad 3587.10 .1038 / \mathrm{s} 41467-021-23942-\mathrm{y}$. 
Saunders, K.O., Lee, E., Parks, R., Martinez, D.R., Li, D., Chen, H., Edwards, R.J., Gobeil, S., Barr, M.,

848 Mansouri, K., et al. (2021). Neutralizing antibody vaccine for pandemic and pre-emergent coronaviruses.

849 Nature 594, 553-559. 10.1038/s41586-021-03594-0.

850 Schmidt, F., Weisblum, Y., Rutkowska, M., Poston, D., DaSilva, J., Zhang, F., Bednarski, E., Cho, A.,

851 Schaefer-Babajew, D.J., Gaebler, C., et al. (2021). High genetic barrier to SARS-CoV-2 polyclonal

852 neutralizing antibody escape. Nature 600, 512-516. 10.1038/s41586-021-04005-0.

853 Smirnov, D., Schmidt, J.J., Capecchi, J.T., and Wightman, P.D. (2011). Vaccine adjuvant activity of 3M-

854 052: an imidazoquinoline designed for local activity without systemic cytokine induction. Vaccine 29,

855 5434-5442. 10.1016/j.vaccine.2011.05.061.

856 Voysey, M., Clemens, S.A.C., Madhi, S.A., Weckx, L.Y., Folegatti, P.M., Aley, P.K., Angus, B., Baillie,

857 V.L., Barnabas, S.L., Bhorat, Q.E., et al. (2021). Safety and efficacy of the ChAdOx1 nCoV-19 vaccine

858 (AZD1222) against SARS-CoV-2: an interim analysis of four randomised controlled trials in Brazil,

859 South Africa, and the UK. Lancet 397, 99-111. 10.1016/S0140-6736(20)32661-1.

860 Wang, L., and Cheng, G. (2021). Sequence analysis of the emerging SARS-CoV-2 variant Omicron in

861 South Africa. J Med Virol. 10.1002/jmv.27516.

862 Wolfel, R., Corman, V.M., Guggemos, W., Seilmaier, M., Zange, S., Muller, M.A., Niemeyer, D., Jones,

863 T.C., Vollmar, P., Rothe, C., et al. (2020). Virological assessment of hospitalized patients with COVID-

864 2019. Nature 581, 465-469. 10.1038/s41586-020-2196-x.

865 Wrapp, D., Wang, N., Corbett, K.S., Goldsmith, J.A., Hsieh, C.L., Abiona, O., Graham, B.S., and

866 McLellan, J.S. (2020). Cryo-EM structure of the 2019-nCoV spike in the prefusion conformation. Science

$867367,1260-1263.10 .1126 /$ science.abb2507.

868 Yang, J., Wang, W., Chen, Z., Lu, S., Yang, F., Bi, Z., Bao, L., Mo, F., Li, X., Huang, Y., et al. (2020). A

869 vaccine targeting the RBD of the S protein of SARS-CoV-2 induces protective immunity. Nature 586 ,

$870 \quad 572-577.10 .1038 / \mathrm{s} 41586-020-2599-8$. 
871 Yu, J., Tostanoski, L.H., Peter, L., Mercado, N.B., McMahan, K., Mahrokhian, S.H., Nkolola, J.P., Liu,

872 J., Li, Z., Chandrashekar, A., et al. (2020). DNA vaccine protection against SARS-CoV-2 in rhesus

873 macaques. Science 369, 806-811. 10.1126/science.abc6284.

874 Zhou, T., Teng, I.T., Olia, A.S., Cerutti, G., Gorman, J., Nazzari, A., Shi, W., Tsybovsky, Y., Wang, L.,

875 Wang, S., et al. (2020). Structure-Based Design with Tag-Based Purification and In-Process Biotinylation

876 Enable Streamlined Development of SARS-CoV-2 Spike Molecular Probes. Cell Rep 33, 108322.

877 10.1016/j.celrep.2020.108322.

878 Zivanov, J., Nakane, T., Forsberg, B.O., Kimanius, D., Hagen, W.J., Lindahl, E., and Scheres, S.H.

879 (2018). New tools for automated high-resolution cryo-EM structure determination in RELION-3. Elife 7.

$880 \quad$ 10.7554/eLife.42166.

881 
A
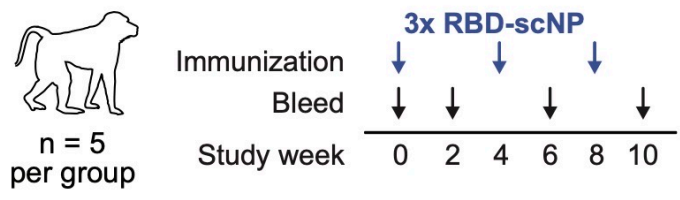

B
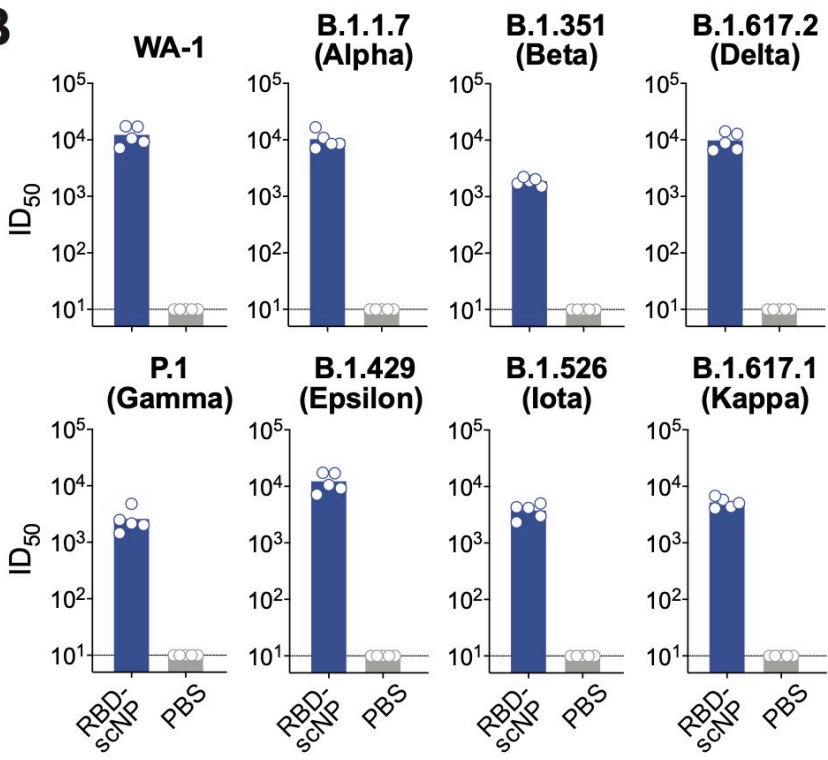

B.1.617.1

Immunization group
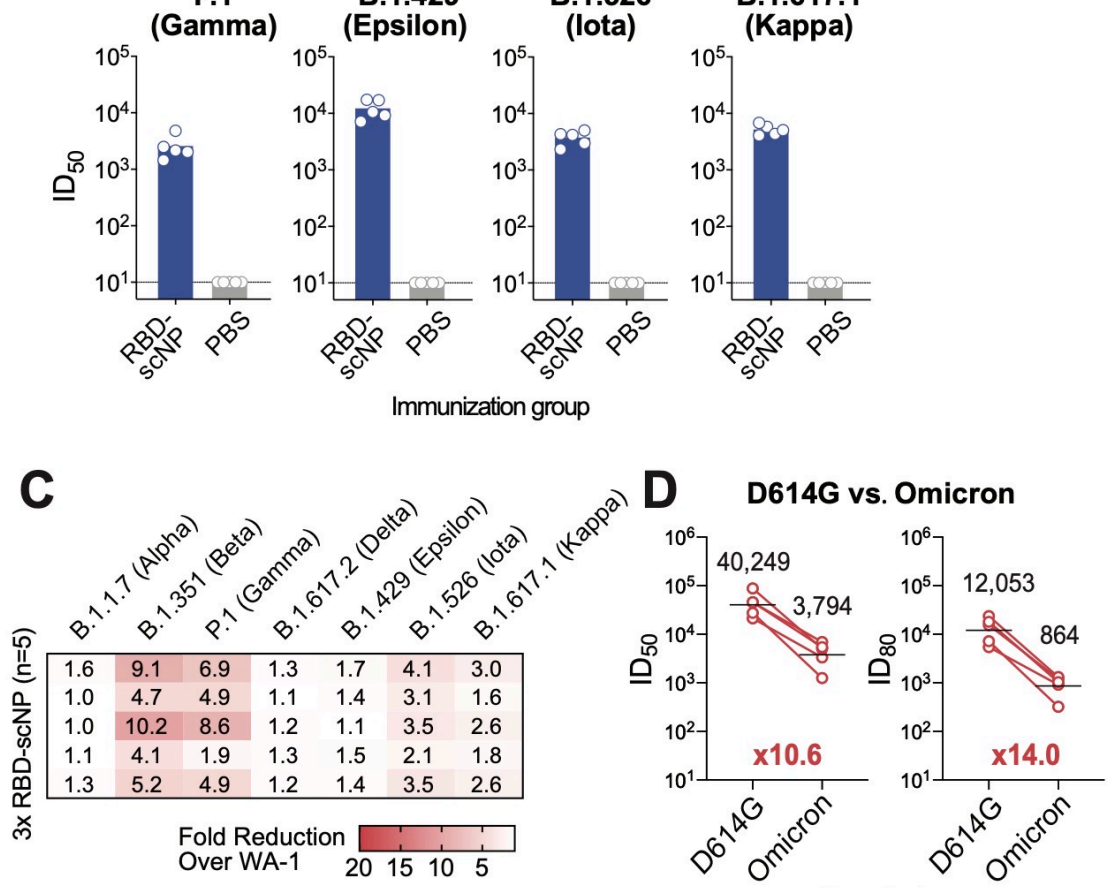

Pseudovirus

Figure 1. RBD-scNP vaccination elicits broad neutralizing antibodies against SARS-CoV-2 variants in macaques.

(A) Schematic of the vaccination study. Cynomolgus macaques ( $n=5$ per group) were immunized 3 times with PBS control or $100 \mu \mathrm{g}$ RBD-scNP adjuvanted with 3M-052-AF + Alum.

(B-C) Plasma antibody (post-3 ${ }^{\text {rd }}$ immunization) neutralization of SARS-CoV-2 variants pseudovirus infection in 293T-ACE2-TMPRSS2 cells. (B) Neutralization $50 \%$ inhibitory dilution $\left(\right.$ ID $\left._{50}\right)$ titers. Each symbol represents an individual macaque. Bars indicate group geometric mean $I_{50}$. (C) Reduction of $I D_{50}$ titers against variants were shown as fold reduction compared to the titers against WA-1.

Each row shows values for an individual macaque for each virus.

(D) Plasma antibody (post-3 ${ }^{\text {rd }}$ immunization) neutralization titers against pseudoviruses of the

SARS-CoV-2 Omicron variants in 293T-ACE2 cells. The geometric mean ID ${ }_{50}$ and $I D_{80}$ titers and the fold reduction compared to D614G are shown. 
(which was not certified by peer review) is the author/funder, who has granted bioRxiv a license to display the preprint in perpetuity. It is $m$ available under aCC-BY-NC-ND 4.0 International license.

Figure 2
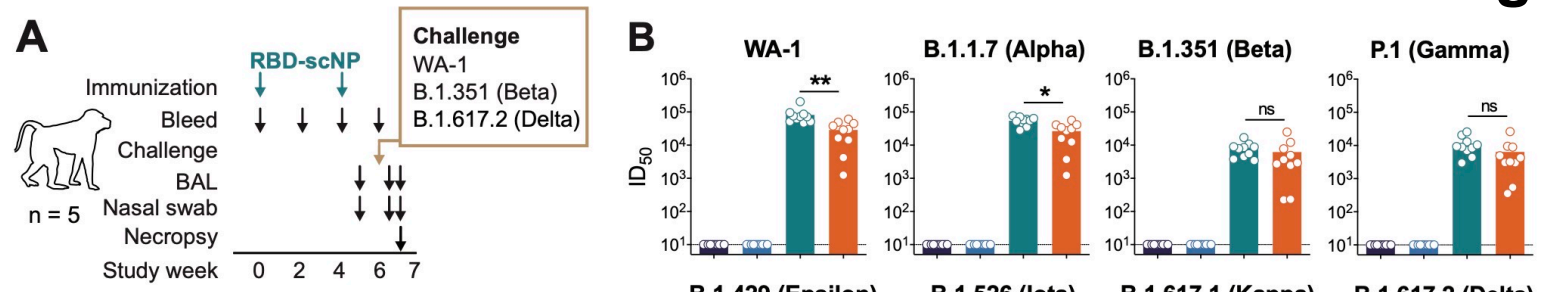

C

B.1.429 (Epsilon) B.1.526 (lota) B.1.617.1 (Kappa) B.1.617.2 (Delta)
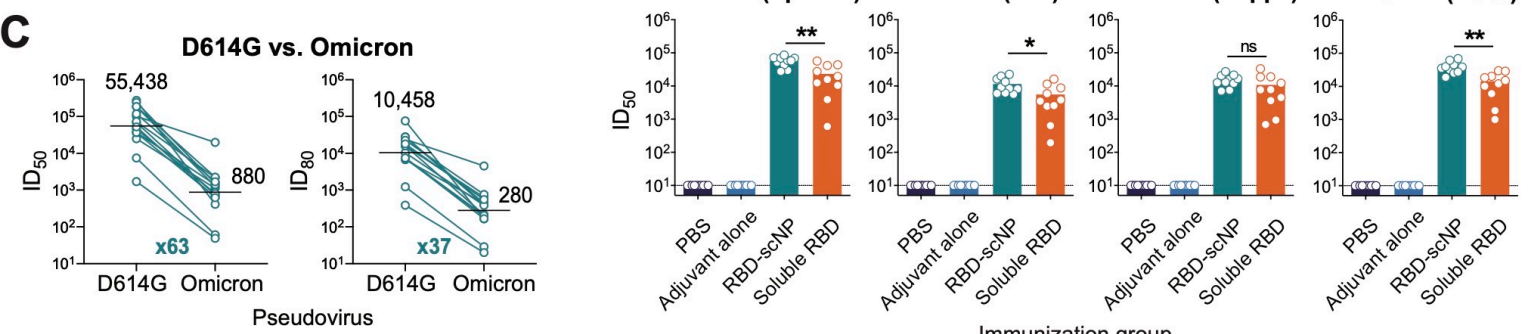

D

WA-1 challenge

E

$$
\text { Immunization group }
$$
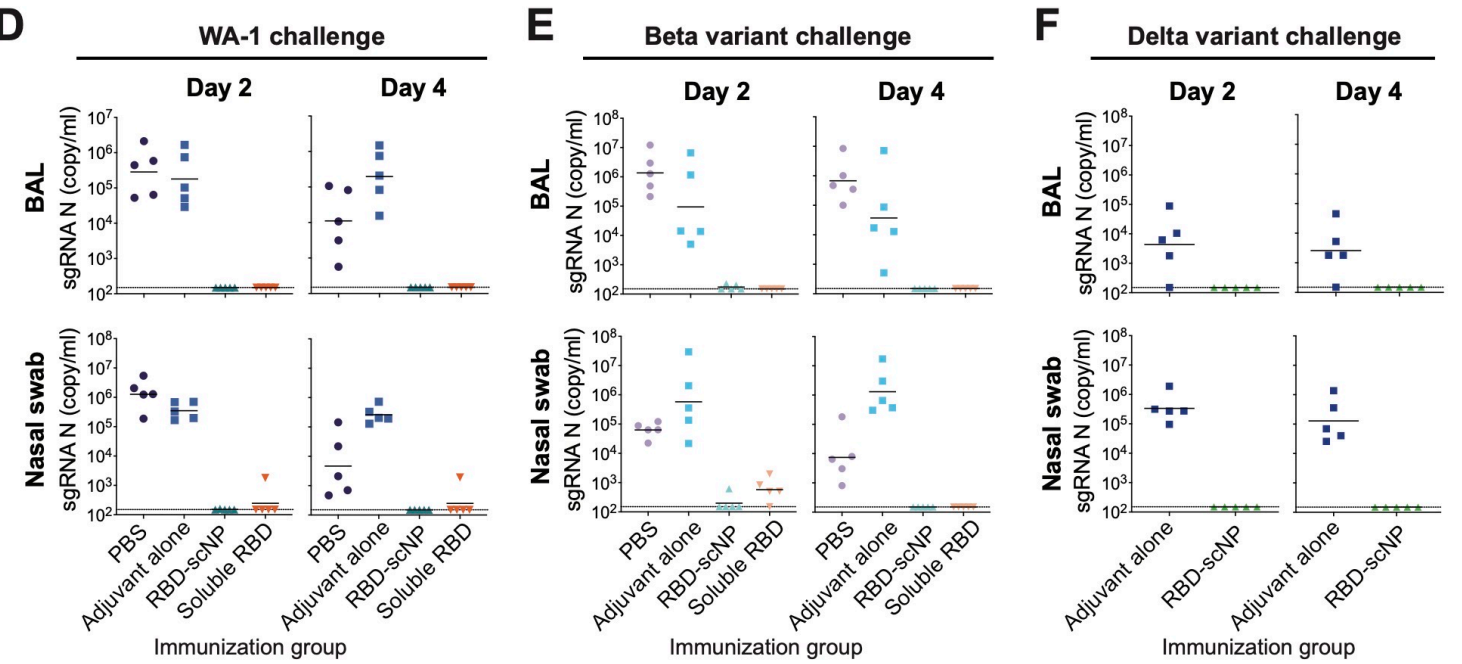

G
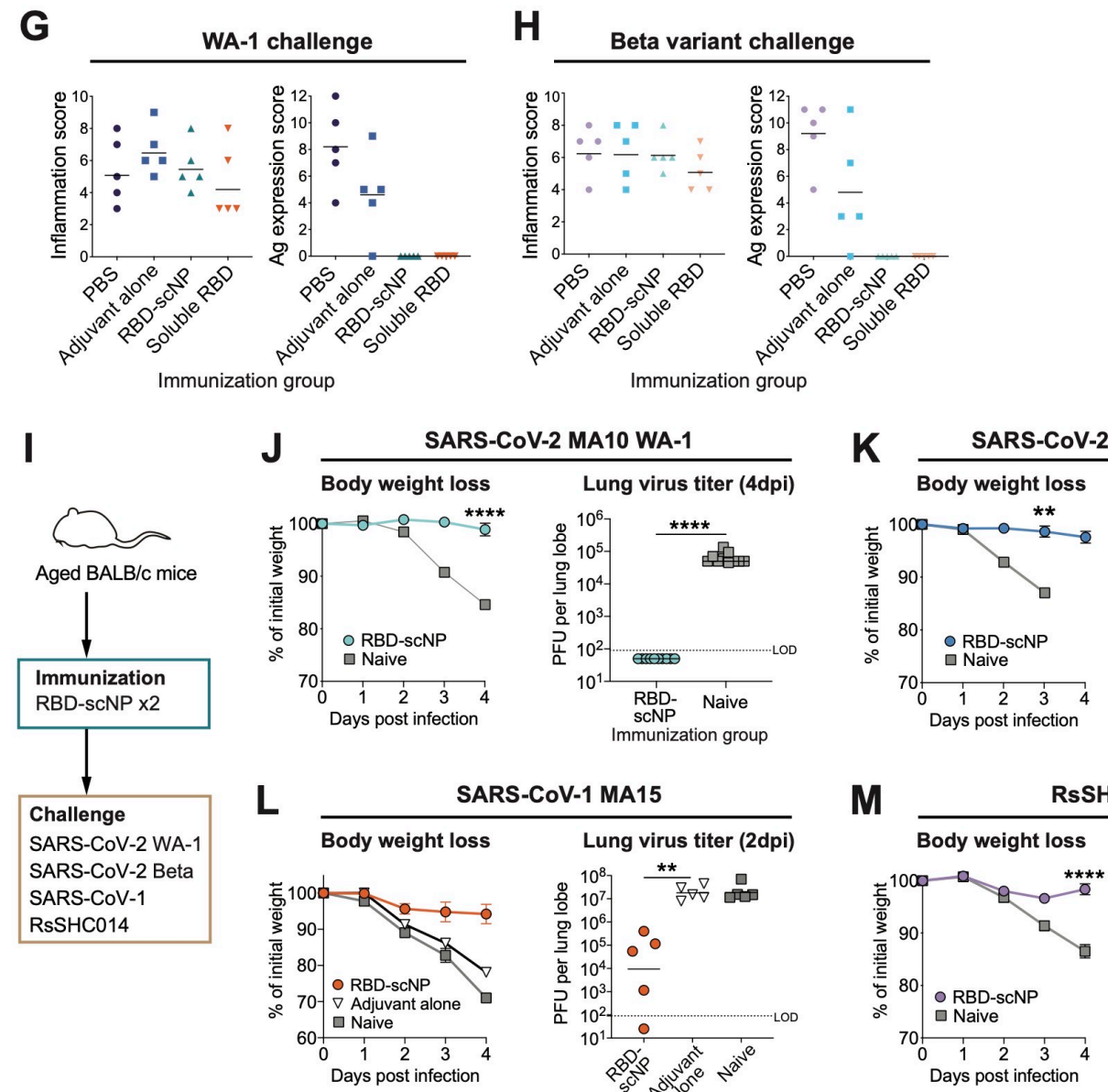

SARS-CoV-1 MA15

M

RsSHC014 MA15
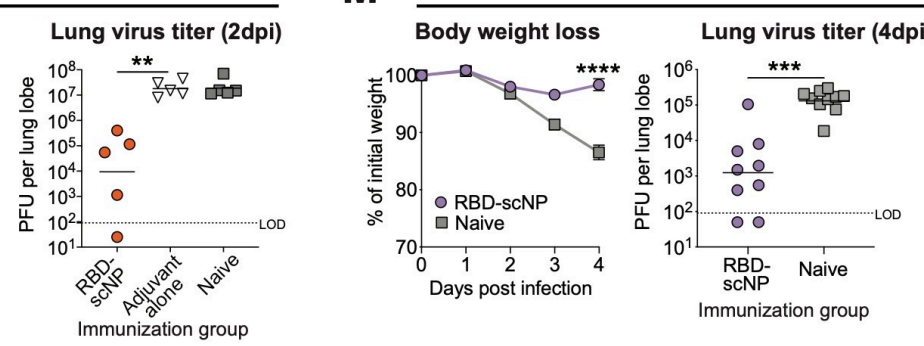
Figure 2. Two doses of RBD-scNP vaccination protected non-human primates and mice from challenges of SARS-CoV-2 variants and other betacoronaviruses.

(A) Schematic of the vaccination and challenge studies. Cynomolgus macaques were immunized twice and challenged with SARS-CoV-2 WA-1 strain $(n=5)$ or B.1.351 (Beta; $n=5)$ or B.1.617.2 (Delta; $n=5)$. Bronchoalveolar lavage (BAL) and nasal swab samples were collected for subgenomic (sgRNA) viral replication tests. Animals were necropsied on day 4 post-challenge for pathologic analysis.

(B) Plasma antibody neutralization ID $_{50}$ titers against pseudoviruses of SARS-CoV-2 variants in 293T-ACE2-TMPRSS2 cells.

(C) Plasma antibody neutralization $I_{50}$ titers against pseudoviruses of SARS-CoV-2 Omicron variants in 293T-ACE2 cells. The geometric mean $I D_{50}$ and $I D_{80}$ titers and the fold reduction compared to D614G are shown.

(D-F) SARS-CoV-2 sgRNA levels for nucleocapsid (N) gene in BAL and nasal swab samples collected on day 2 and 4 after SARS-CoV-2 WA-1 (D), Beta variant (E) or Delta variant (F) challenge. Dashed line indicates limit of the detection (LOD).

(G-H) Histopathological analysis of the SARS-CoV-2 WA-1 (G) and Beta variant $(\mathrm{H})$ challenged monkeys. Scores of lung inflammation determined by haematoxylin and eosin (H\&E) staining and SARS-CoV-2 nucleocapsid antigen (Ag) expression determined by immunohistochemistry (IHC) staining.

(I) Schematic of the mouse challenge studies. Aged female BALB/c mice ( $n=10$ per group) were immunized intramuscularly twice and challenged with SARS-CoV-2 mouse-adapted 10 (MA10) WA-1, SARS-CoV-2 MA10 Beta variant, SARS-CoV-1 mouse-adapted 15 (MA15), or Bat coronavirus (CoV) RsSHC014 MA15.

(J) Weight loss and lung virus titers at 4 days post-infection (dpi) of the SARS-CoV-2 MA10 WA-1 challenged mice.

(K) Weight loss and lung virus titers at 2 dpi of the SARS-CoV-2 MA10 Beta variant challenged mice.

(L) Weight loss and lung virus titers at 2 dpi of the SARS-CoV-1 MA15 challenged mice.

(M) Weight loss and lung virus titers at 4 dpi of the Bat CoV RsSHC014 MA15 challenged mice. ns, not significant, ${ }^{*} \mathrm{P}<0.05$, ${ }^{* *} \mathrm{P}<0.01,{ }^{* * *} \mathrm{P}<0.001$, ${ }^{* * *} \mathrm{P}<0.0001$, Wilcoxon rank sum exact test. 
A

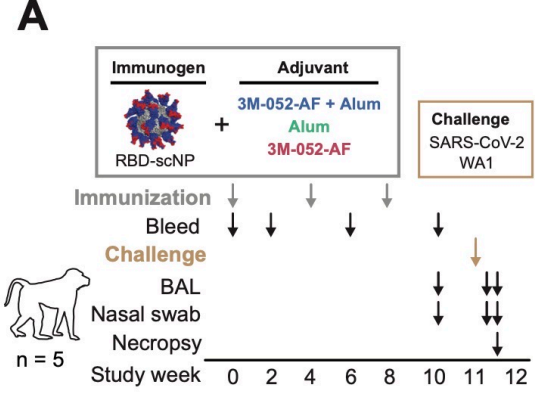

B

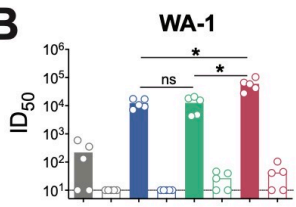

B.1.429 (Epsilon)

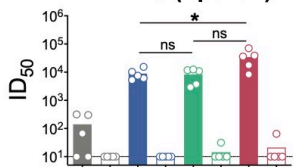

mmunogen:

C

D614G vs. Omicron

- RBD-scNP / 3M-052-AF + Alum

- RBD-scNP / Alum

- RBD-scNP / 3M-052-AF
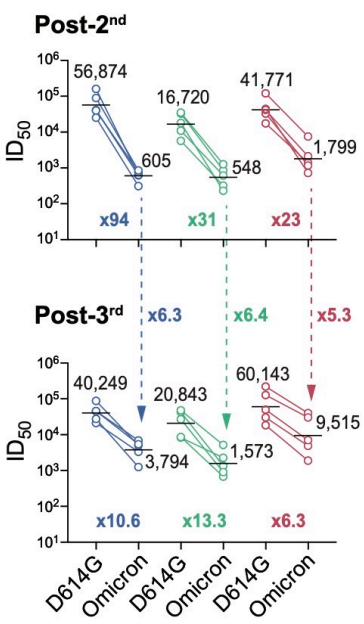

Pseudovirus

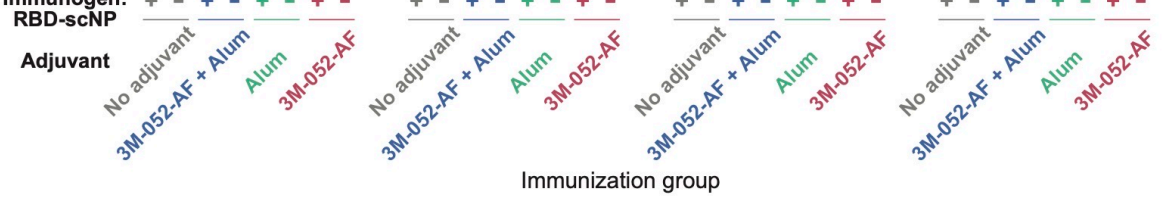

D
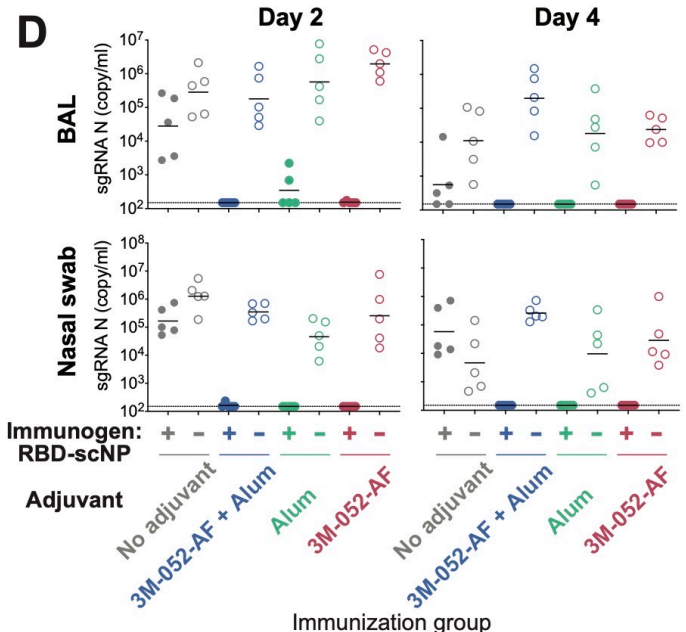

E
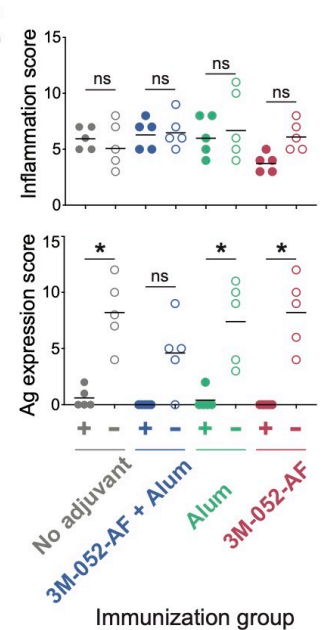

Figure 3. Neutralizing antibodies and in vivo protection elicited by RBD-scNP vaccine formulated with three different adjuvants.

(A) Schematic of the vaccination and challenge study. Cynomolgus macaques ( $n=5$ per group) were immunized intramuscularly 3 times with $100 \mu \mathrm{g}$ of RBD-scNP adjuvanted with 3M052-AF + Alum, Alum, 3M052-AF, or PBS control. Animals injected with adjuvant alone or PBS were set as control groups. Monkeys were then challenged with SARS-CoV-2 WA-1, collected for blood, BAL and nasal swab samples, and necropsied for pathologic analysis.

(B) Neutralization $I_{50}$ titers of plasma antibodies (post-3rd immunization) against pseudovirus of SARS-CoV-2 variants in 293T-ACE2-TMPRSS2.

(C) Plasma antibody (post-2 ${ }^{\text {nd }}$ and post-3rd immunization) neutralization titers against pseudoviruses of the SARS-CoV-2 Omicron variants in 293T-ACE2 cells. The geometric mean ID50 titers and the fold reduction compared to D614G are shown. The dashed arrows indicate fold increase of $I D_{50}$ titer induced by the $3^{\text {rd }}$ boost.

(D) SARS-CoV-2 N gene sgRNA in BAL and nasal swab samples collected on day 2 and 4 postchallenge. Dashed line indicates limit of the detection.

(E) Histopathological analysis. Lung sections from each animal were scored for lung inflammation by H\&E staining, and for SARS-CoV-2 nucleocapsid Ag expression by IHC staining.

ns, not significant, ${ }^{*} \mathrm{P}<0.05$, Wilcoxon rank sum exact test. 
A

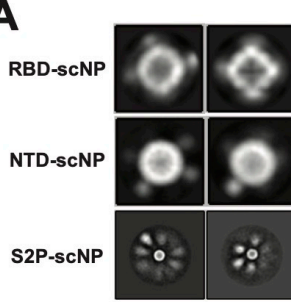

B

\section{E}
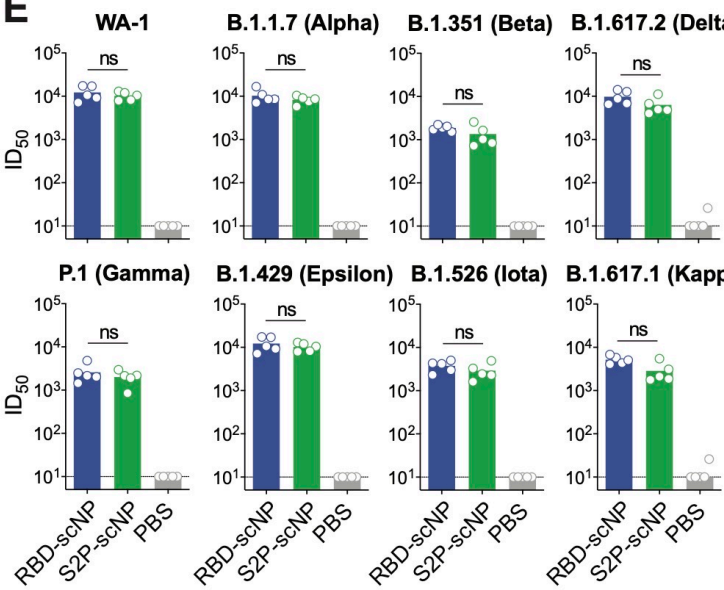

Immunization group
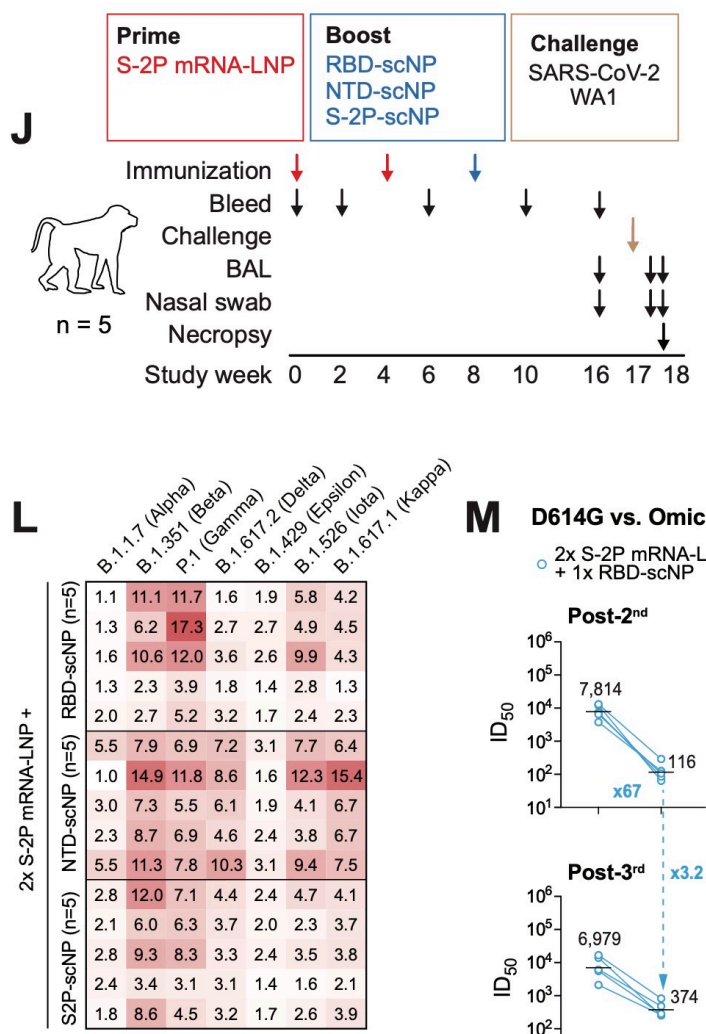

Fold Reduction Over WA-1

$2015 \quad 10 \quad 5$
M D614G vs. Omicron $2 \times$ S-2P mRNA-LNP
+1 RBD-scNP
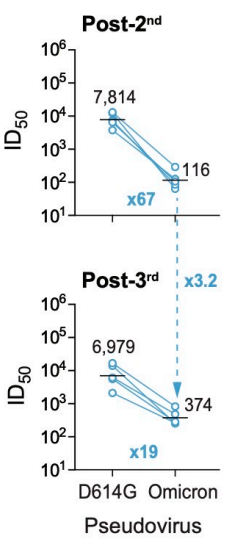

$\mathbf{F}$

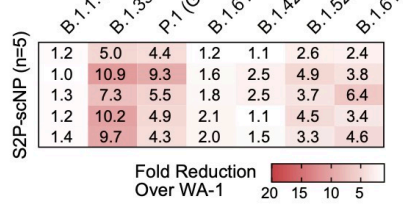

H

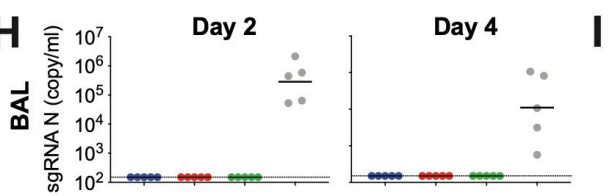

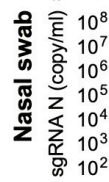

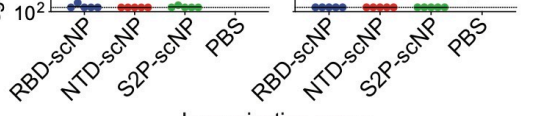

Immunization group

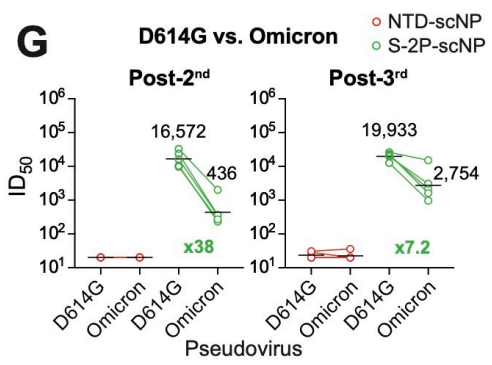

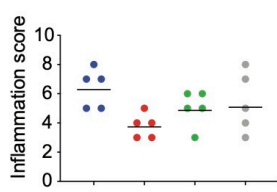

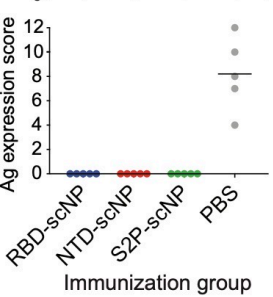

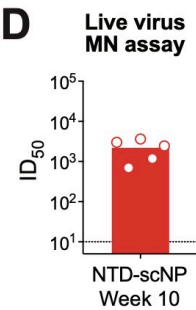

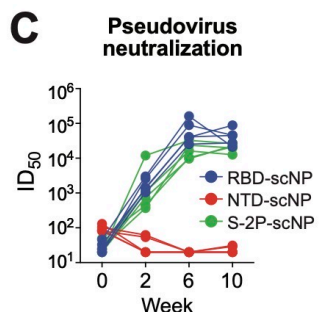

K
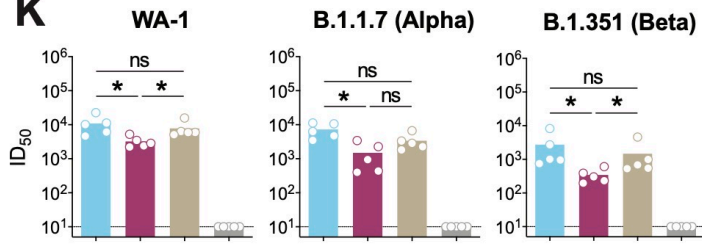

B.1.526 (lota)
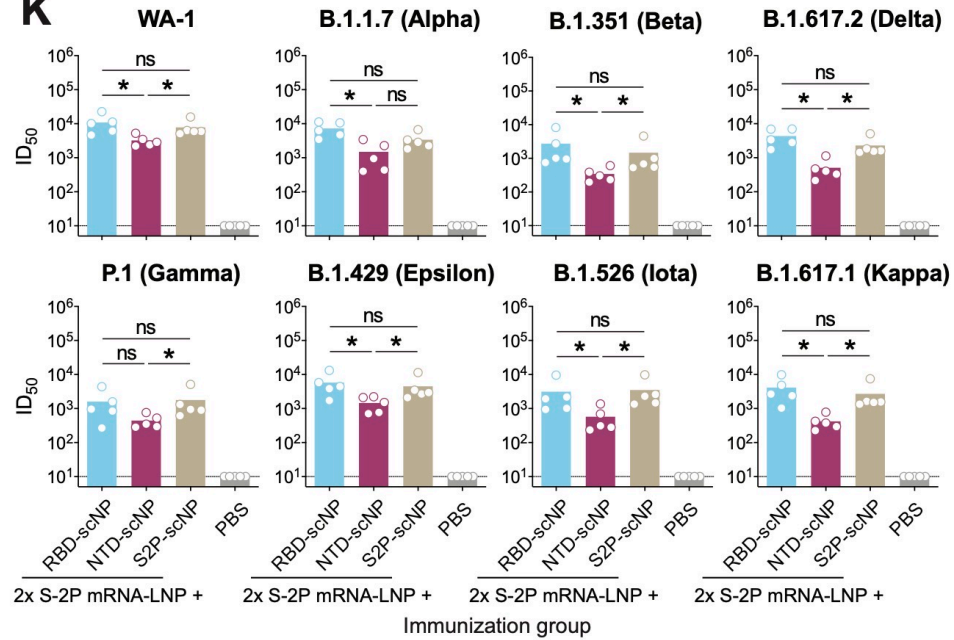

$\mathbf{N}$

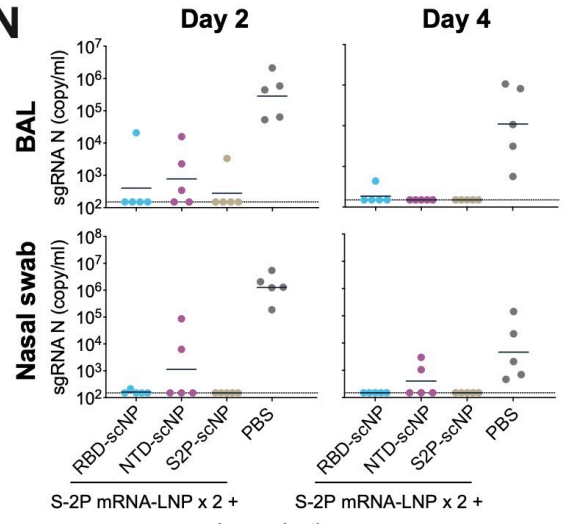

Immunization group

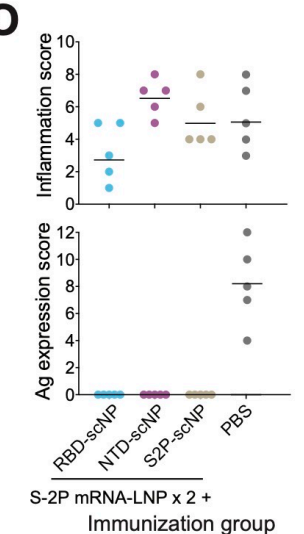


Figure 4. Neutralizing antibodies and in vivo protection induced by RBD-scNP, NTDscNP and S2P-scNP vaccines as a three-dose regimen or as a heterologous boost for S2P mRNA-LNP vaccine.

(A) Negative-stain electron microscopy 2D class averaging of RBD-scNP, NTD-scNP, and S2P-scNP. The size of each box: RBD-scNP and NTD-scNP, $257 \AA$; S2P-scNP, 1,029 $\AA$.

(B) Schematic of the three-dose regimen. Cynomolgus macaques ( $n=5$ per group) were immunized 3 times with RBD-scNP, NTD-scNP, or S2P-scNP adjuvanted with 3M-052-AF + Alum. Monkeys were then challenged with SARS-CoV-2 WA-1, collected for blood, BAL and nasal swab samples, and necropsied for pathologic analysis.

(C) Neutralization ID $_{50}$ of plasma antibodies (week 0, 2, 6 and 10) against pseudotyped SARSCoV-2 D614G strain in 293T/ACE2.MF cells.

(D) Neutralization ID $_{50}$ of the NTD-scNP-induced antibodies (week 10) against live SARS-CoV2 WA-1 virus in Vero-E6 cells in microneutralization (MN) assay.

(E-F) Plasma antibody neutralization against pseudoviruses of the SARS-CoV-2 variants in 293T-ACE2-TMPRSS2 cells. (E) ID $D_{50}$ titers and (F) reduction of $I D_{50}$ titers against variants were shown as fold reduction compared to the titers against WA-1.

(G) NTD-scNP- or S2P-scNP-induced plasma antibody (post-2 ${ }^{\text {nd }}$ and post-3 $3^{\text {rd }}$ immunization) neutralization titers against pseudoviruses of the SARS-CoV-2 Omicron variants in 293T-ACE2 cells. The geometric mean $I_{50}$ titers and the fold reduction compared to D614G are shown. (H) SARS-CoV-2 N gene sgRNA in BAL and nasal swab samples collected on day 2 and 4 post-challenge.

(I) Histopathological analysis. Scores of lung inflammation determined by H\&E staining and SARS-CoV-2 nucleocapsid antigen expression determined by IHC staining.

(J) Schematic of the heterologous prime-boost regimen. Cynomolgus macaques $(n=5$ per group) were immunized 2 times with S2P mRNA-LNP, and boosted with adjuvanted RBDscNP, NTD-scNP, or S2P-scNP vaccine. Monkeys were then challenged with SARS-CoV-2 WA-1, collected for blood, BAL and nasal swab samples, and necropsied for pathologic analysis.

(K-L) Plasma antibody neutralization against pseudoviruses of SARS-CoV-2 variants in 293TACE2-TMPRSS2 cells. (K) ID $I_{50}$ titers. (L) Reduction of $I D_{50}$ titers against variants were shown as fold reduction compared to the titers against WA-1.

(M) Plasma antibody (post-2 ${ }^{\text {nd }}$ and post- $3^{\text {rd }}$ immunization) neutralization titers against pseudoviruses of the SARS-CoV-2 Omicron variants in 293T-ACE2 cells. The geometric mean $\mathrm{ID}_{50}$ titers and the fold reduction compared to $\mathrm{D} 614 \mathrm{G}$ are shown. The dashed arrow indicates fold increase of $I D_{50}$ titer induced by the $3^{\text {rd }}$ boost.

(N) SARS-CoV-2 N gene sgRNA in BAL and nasal swab samples collected on day 2 and 4 post-challenge.

(O) Histopathological analysis. Scores of lung inflammation determined by H\&E staining and SARS-CoV-2 nucleocapsid antigen expression determined by IHC staining.

ns, not significant, ${ }^{*} \mathrm{P}<0.05$, Wilcoxon rank sum exact test. 


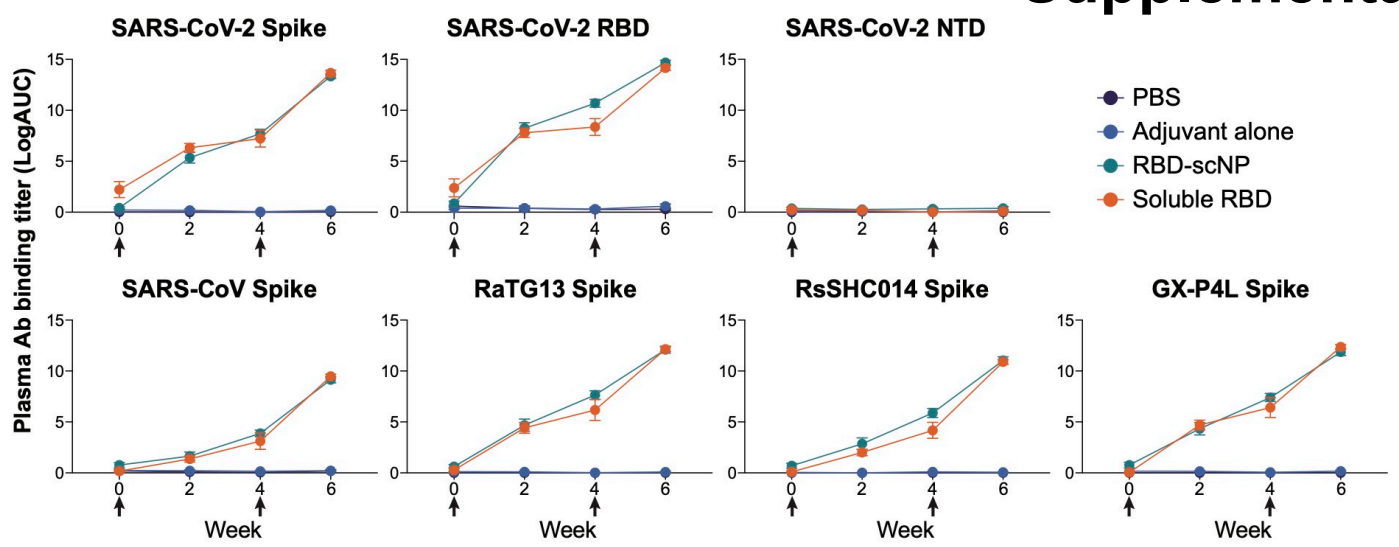

B

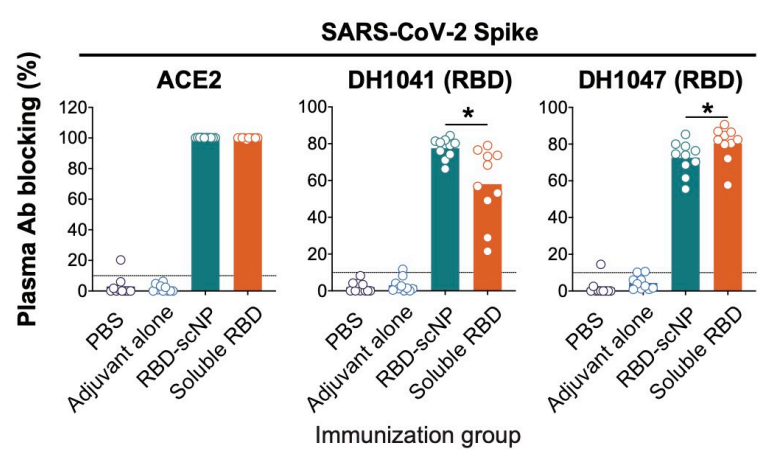

C

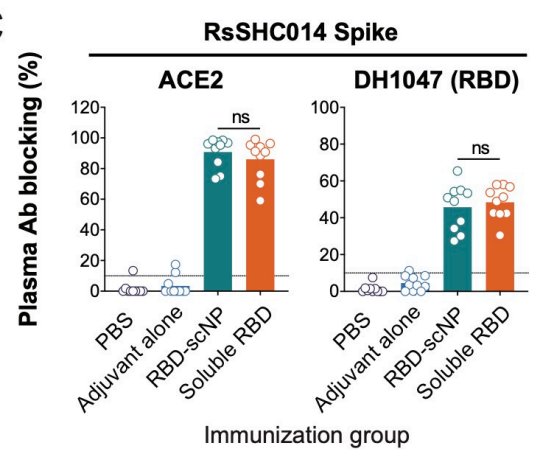

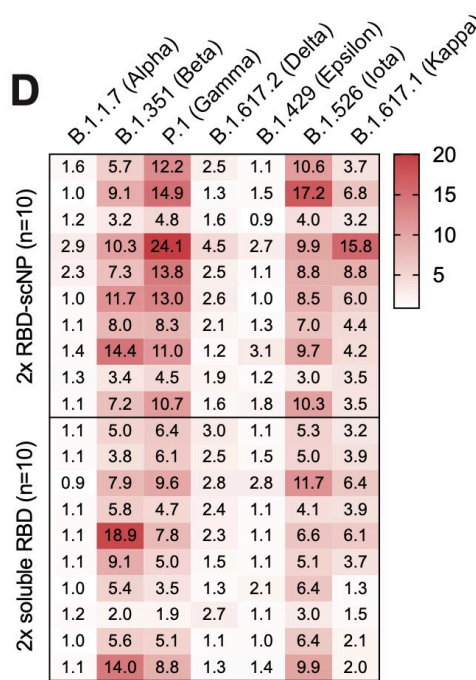
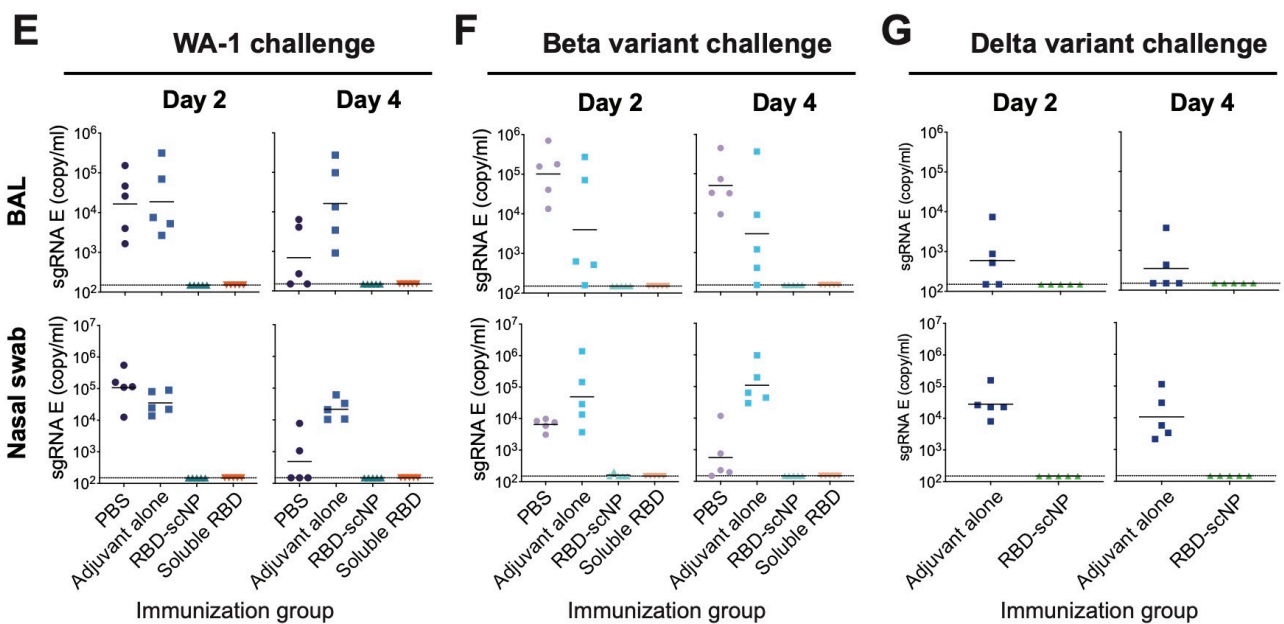

Supplementary Figure 1. RBD-scNP elicited higher titers of neutralizing antibodies than soluble RBD. Related to Figure 2.

(A) Plasma antibody binding titers to SARS-CoV-2 spike, RBD and NTD, as well as recombinant spike proteins of SARS-CoV, bat CoVs RaTG13, RsSHC014, and pangolin CoV GX-P4L. ELISA binding titers are shown as mean \pm SEM of log area-under-curve (AUC).

(B-C) Plasma antibody (post-2 ${ }^{\text {nd }}$ immunization) blocking activity. ELISA was performed to test plasma antibodies blocking ACE2, human RBD neutralizing antibodies DH1041 and DH1047 binding to SARS-CoV-2 spike protein (B), or blocking ACE2 and DH1047 binding to RsSHC014 spike protein (C). Data are expressed as \% blocking of ACE or the indicated antibody by 1:50 diluted plasma samples.

(D) Fold reduction of plasma antibody $I_{50}$ titers against pseudoviruses of SARS-CoV-2 variants in 293T-ACE2-TMPRSS2 cells, compared to the titers against WA-1.

(E-G) SARS-CoV-2 E gene sgRNA in BAL and nasal swab samples from the WA-1 (E), Beta variant $(F)$, and Delta variant $(G)$ challenged monkeys. Dashed line indicates limit of the detection.

ns, not significant, ${ }^{*} \mathrm{P}<0.05$, Wilcoxon rank sum exact test. 
A
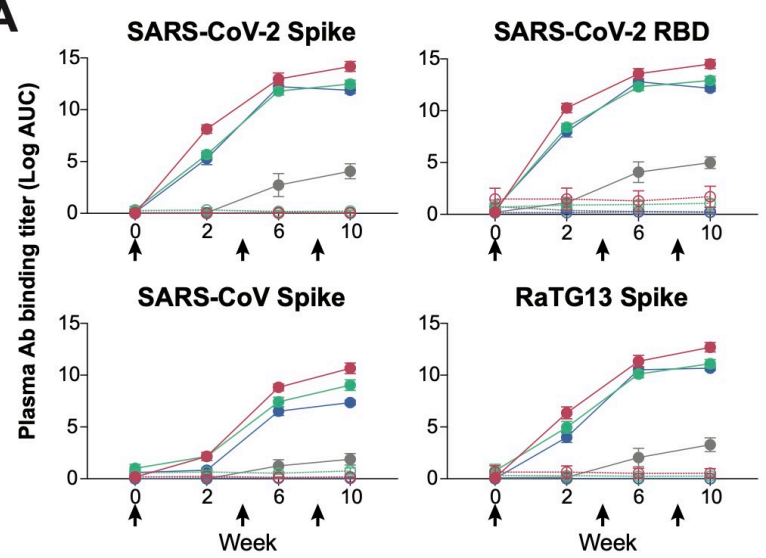

RaTG13 Spike

B

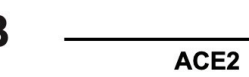

SARS-CoV-2 Spike DH1041 (RBD)

Immunogen: + - + - + - +

Adjuvant

D

\section{SARS-CoV-2 Spike}
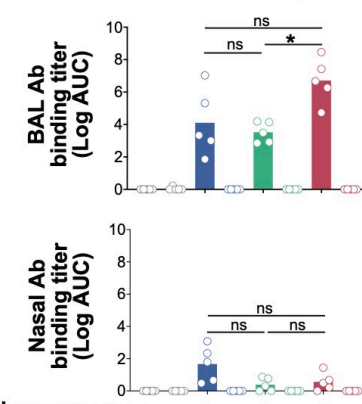

Immunogen: + - + + + + RBD-scNP

Adjuvant 30

Immunization group

$\mathbf{F}$

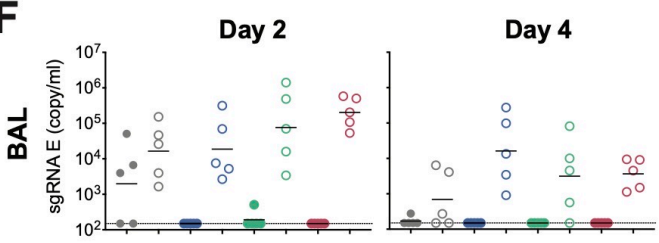

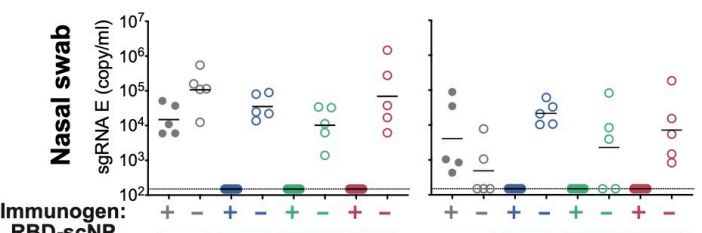

SARS-CoV-2 RBD
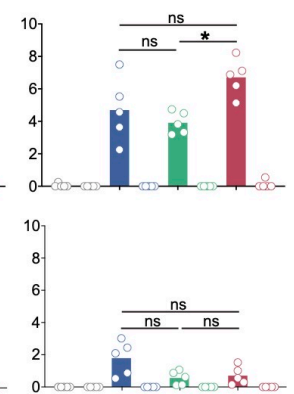

$+-+-++-$

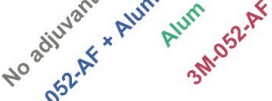

3 group
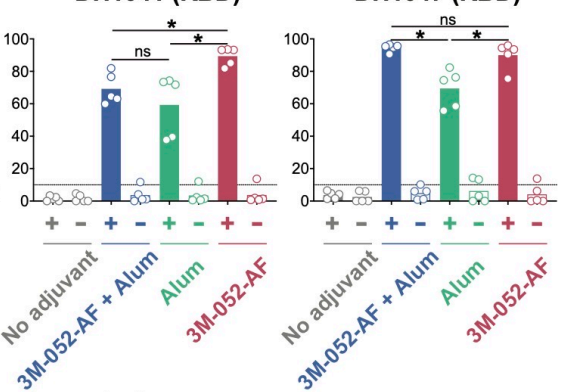

E
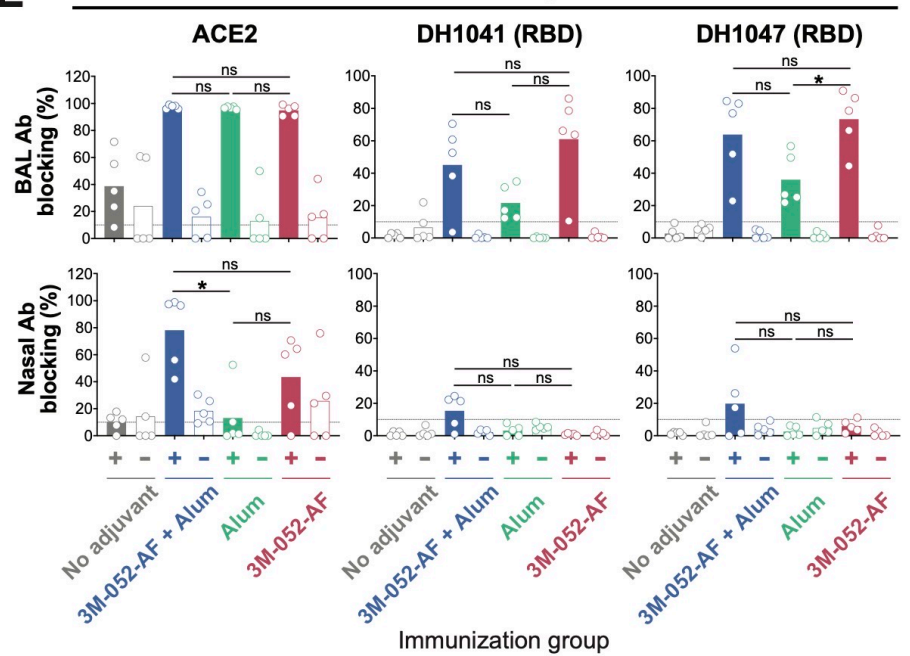

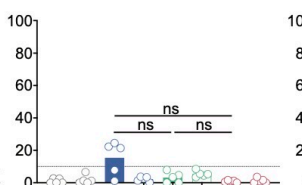

RsSHC014 Spike
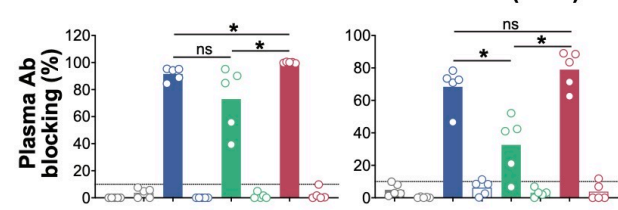

RBD-scNP

Adjuvant

Immunization group

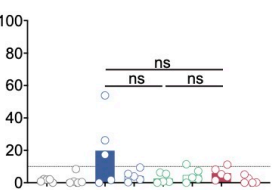

+ No adjuvant

\begin{tabular}{l|l}
+ & $3 \mathrm{M}-052-\mathrm{AF}+$ Alum
\end{tabular}

+ Alum

GX-P4L Spike

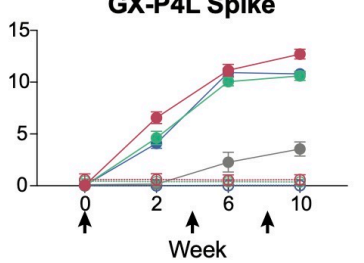




\section{Supplementary Figure 2. Serum and mucosal antibody responses elicited by RBD-scNP} formulated with three different adjuvants. Related to Figure 3.

(A) Plasma antibody binding titers to SARS-CoV-2 spike, RBD and NTD, as well as recombinant spike proteins of SARS-CoV, bat CoV RaTG13, RsSHC014, and pangolin CoV GX-P4L. ELISA binding titers are shown as mean \pm SEM of log area-under-curve (AUC).

(B-C) Plasma antibody (post-2 ${ }^{\text {nd }}$ immunization) blocking activity. ELISA was performed to test plasma antibodies blocking ACE2, human RBD neutralizing antibodies DH1041 and DH1047 binding to SARS-CoV-2 spike protein (B), or blocking ACE2 and DH1047 binding to RsSHC014 spike protein (C). Data are expressed as \% blocking of ACE or the indicated antibody by 1:50 diluted plasma samples.

(D-E) Mucosal antibody binding and blocking activities after the $3^{\text {rd }}$ immunization. ELISA was performed to test 10x concentrated BAL or unconcentrated nasal wash samples binding to SARSCoV-2 spike and RBD (D), or blocking ACE2, DH1041 and DH1047 binding to SARS-CoV-2 spike protein (E). Binding titers are expressed as log AUC, and blocking activities are shown as \%blocking of ACE or the indicated antibody.

(F) SARS-CoV-2 E gene sgRNA in BAL and nasal swab samples from the WA-1 challenged monkeys. Dashed line indicates limit of the detection.

ns, not significant, ${ }^{*} \mathrm{P}<0.05$, Wilcoxon rank sum exact test. 
A

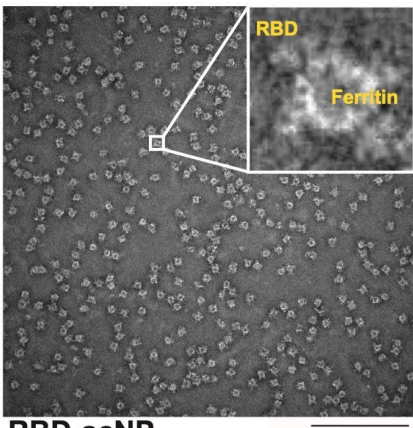

B

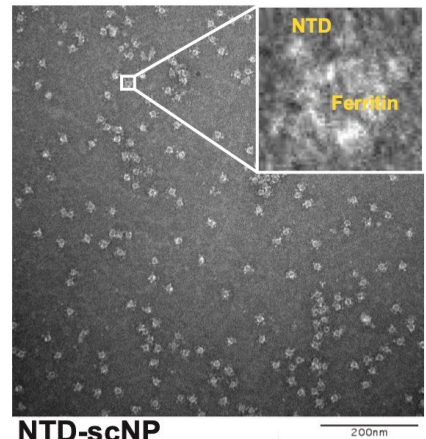

NTD-scNP
C

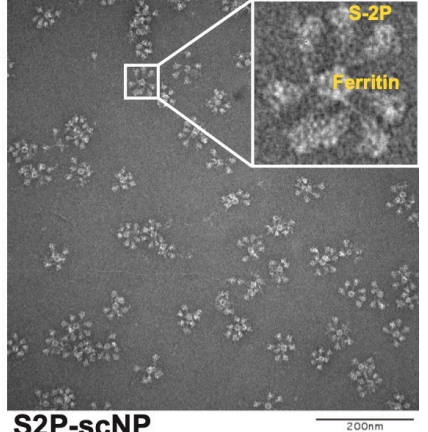

D
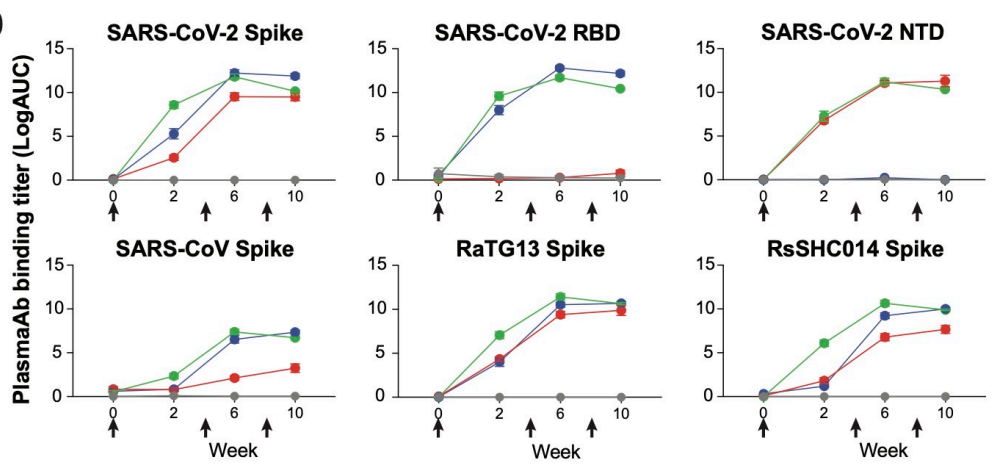

E

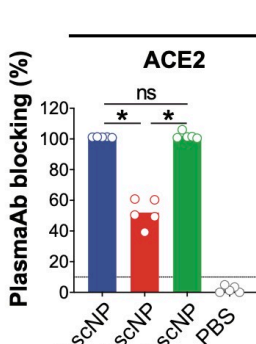

SARS-CoV-2 Spike
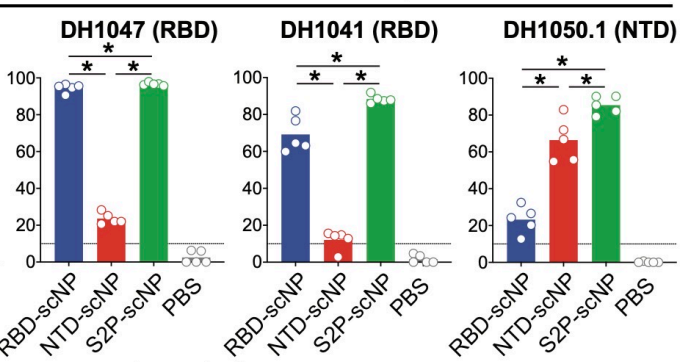

Immunization group

G

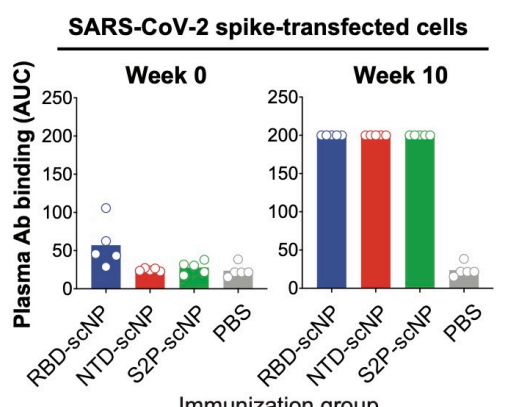

Immunization group
H

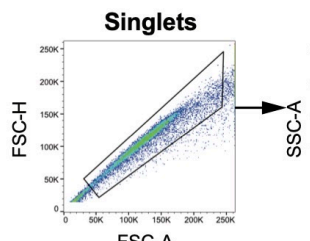

RBD-scNP

- NTD-scNP

- S-2P-scNP

- PBS

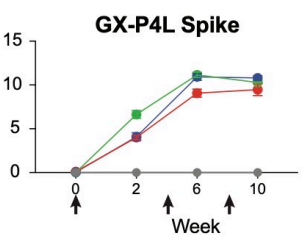

$\mathbf{F}$

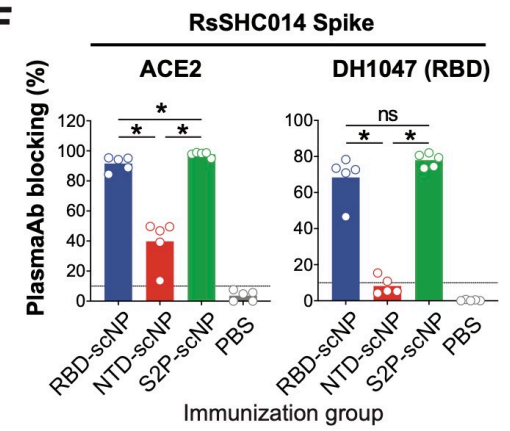

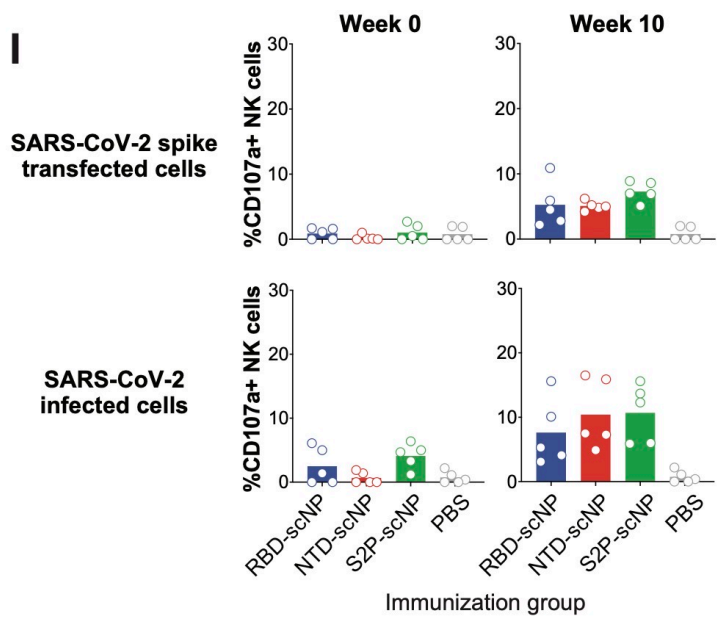

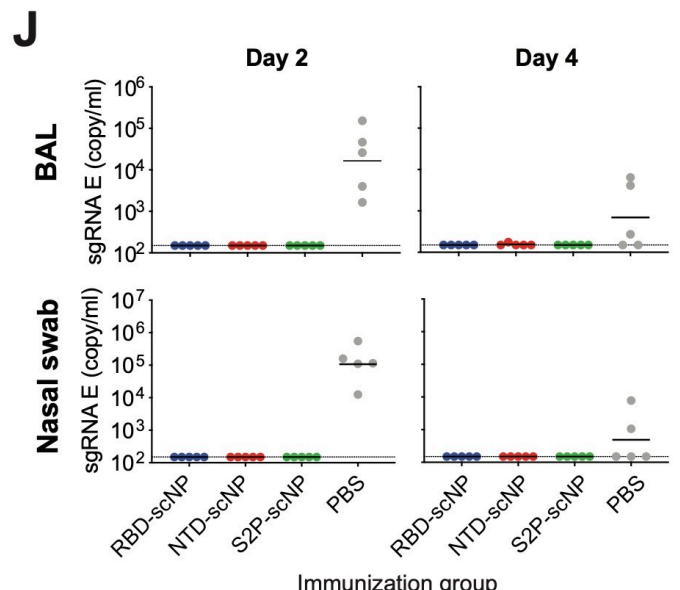




\section{Supplementary Figure 3. RBD-scNP, NTD-scNP and S2P-scNP induced spike-binding} antibodies and mediated antibody-dependent cellular cytotoxicity (ADCC). Related to Figure 4.

(A-C) Negative stain electron microscopy imaging of RBD-scNP (A), NTD-scNP (B), and S2PscNP (C). The inset shows zoomed-in image of representative scNPs.

(D) Plasma antibody binding titers to SARS-CoV-2 spike, RBD and NTD, as well as recombinant spike proteins of SARS-CoV, bat CoV RaTG13, RsSHC014, and pangolin CoV GX-P4L. ELISA binding titers are shown as mean \pm SEM of log area-under-curve (AUC).

(E-F) Plasma antibody (post-2 ${ }^{\text {nd }}$ immunization) blocking activity. ELISA was performed to test plasma antibodies blocking ACE2, human RBD neutralizing antibodies DH1041 and DH1047, human NTD neutralizing antibodies DH1050.1 binding to SARS-CoV-2 spike protein (E), or blocking ACE2 and DH1047 binding to RsSHC014 spike protein (F). Data are expressed as \% blocking of ACE or the indicated antibody by 1:50 diluted plasma samples.

(G) Pre-immunization (week 0) and pre-challenge (week 10, post-3rd immunization) plasma antibodies binding on SARS-CoV-2 spike-transfected 293T cells tested by cell surface staining.

(H) The gating strategy for the NK cell degranulation ADCC assay. Purified human NK cells were mixed with SARS-CoV-2 spike-transfected cells or SARS-CoV-2 infected cells in the presence of 1:50 diluted plasma samples. NK cell degranulation was detected based on CD107a expression. (I) RBD-scNP-, NTD-scNP- and S2P-scNP-induced antibodies mediated ADCC. The percentages of CD107a+ NK cells were shown when NK cells were assayed with plasma antibodies (week 0 and week 10) in SARS-CoV-2 spike transfected 293T cells (top row) or SARS-CoV-2 infected Vero E6 cells cells (botton row).

(J) SARS-CoV-2 E gene sgRNA in BAL and nasal swab samples from WA-1 challenged monkeys. Dashed line indicates limit of the detection. 
A

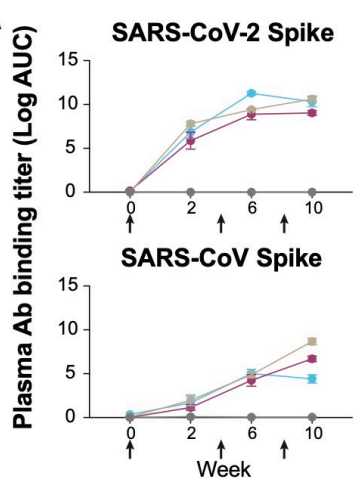

B

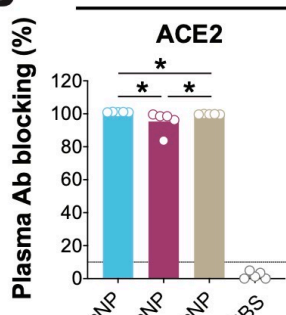

SARS-CoV-2 Spike
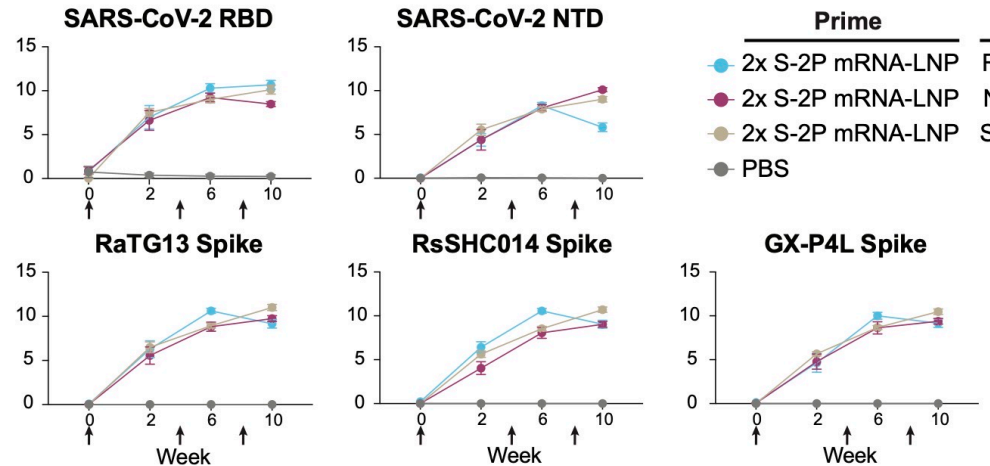

C
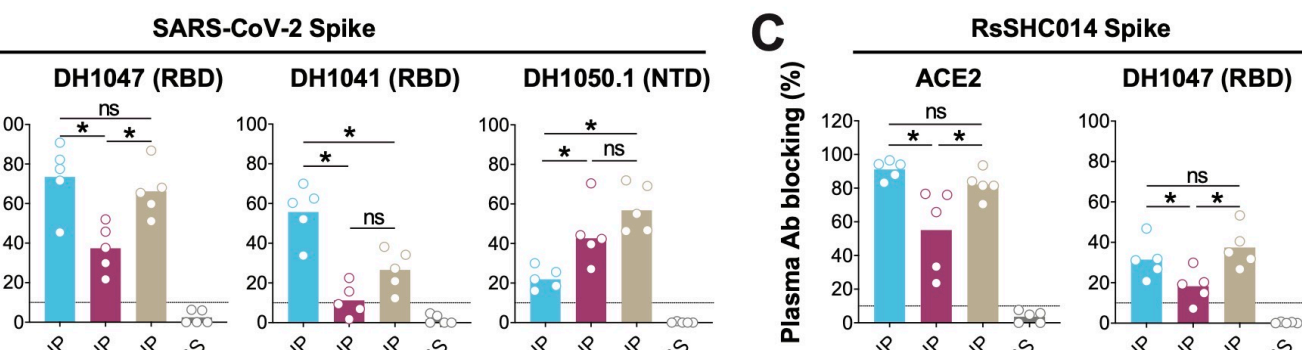

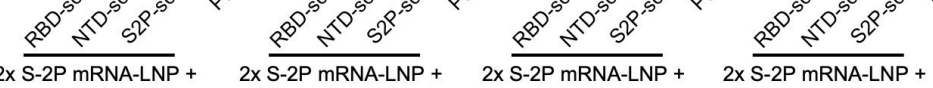
Immunization group

D

ACE2

BAL

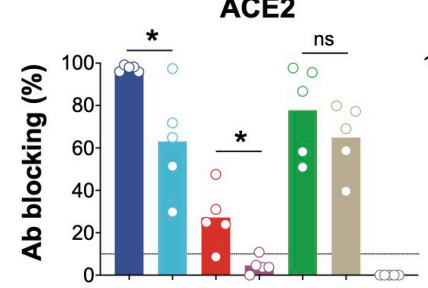

DH1047 (RBD)
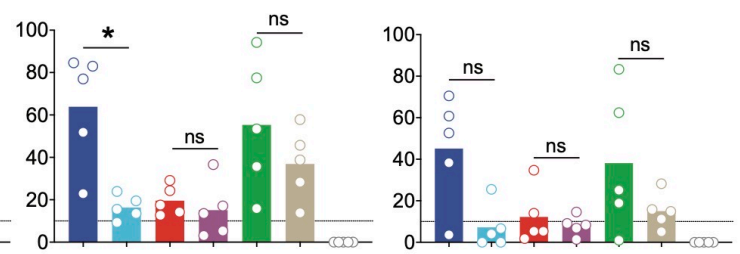

E
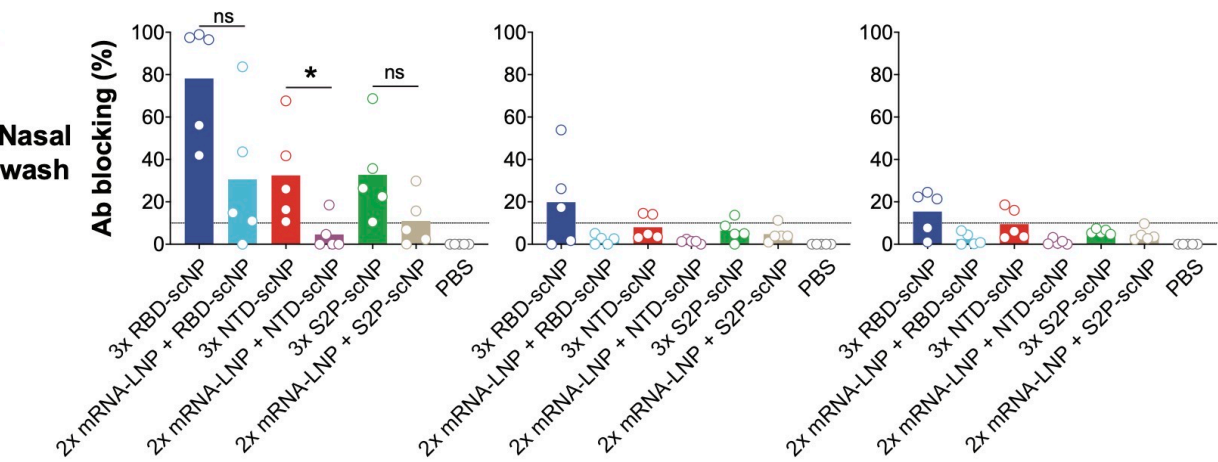

$\mathbf{F}$
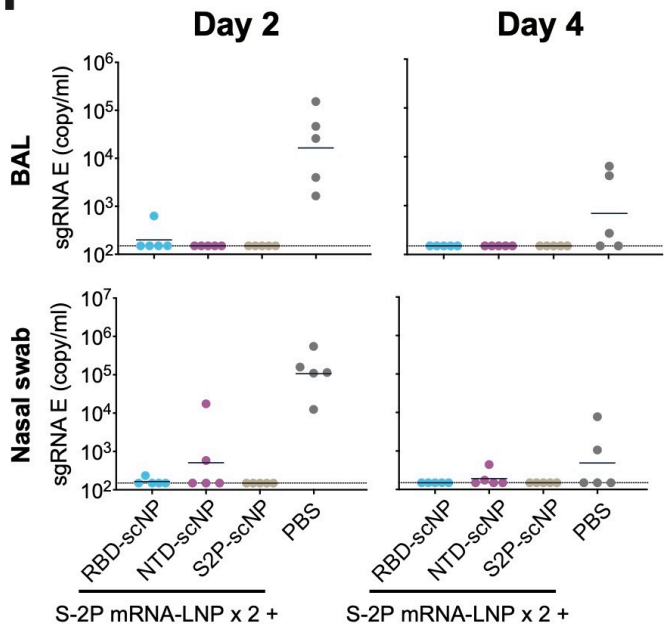

Immunization group 


\section{Supplementary Figure 4. Antibody responses elicited by scNP vaccines as a booster vaccination in macaques that received two doses of S-2P mRNA-LNP vaccine. Related to Figure 4.}

(A) Plasma antibody binding titers to SARS-CoV-2 spike, RBD and NTD, as well as recombinant spike proteins of SARS-CoV, bat CoV RaTG13, RsSHC014, and pangolin CoV GX-P4L. ELISA binding titers are shown as mean \pm SEM of log area-under-curve (AUC). (B-C) Plasma antibody (post-2 ${ }^{\text {nd }}$ immunization) blocking activity. ELISA was performed to test plasma antibodies blocking ACE2, human RBD neutralizing antibodies DH1041 and DH1047, human NTD neutralizing antibodies DH1050.1 binding to SARS-CoV-2 spike protein (B), or blocking ACE2 and DH1047 binding to RsSHC014 spike protein (C). Data are expressed as $\%$ blocking of ACE or the indicated antibody by 1:50 diluted plasma samples.

(D-E) Comparison of mucosal antibody blocking activities induced by 3 doses of scNP vaccination or 2 doses of S2P mRNA-LNP +1 dose of scNP vaccination. ELISA for 10x concentrated BAL samples (D) and neat nasal wash samples $(E)$ blocking the binding of ACE2 or neutralizing antibody (DH1041 or DH1047) on SARS-CoV-2 spike were performed. Data are expressed as \% blocking of ACE or the indicated antibody by mucosal samples. (F) SARS-CoV-2 E gene sgRNA in BAL and nasal swab samples from WA-1 challenged monkeys. Dashed line indicates limit of the detection.

ns, not significant, ${ }^{*} \mathrm{P}<0.05$, Wilcoxon rank sum exact test. 